\title{
An Optimization Design Method of Express Delivery Service Based on Quantitative Kano Model and Fuzzy QFD Model
}

\author{
Hongmei Shan $\left(\mathbb{D},{ }^{1}\right.$ Xinmeng Fan, ${ }^{1}$ Siyu Long, ${ }^{2}$ Xuejing Yang, ${ }^{1}$ and Shuhan Yang ${ }^{3}$ \\ ${ }^{1}$ Department of Logistics Management College of Modern Posts, Xi'an University of Posts and Telecommunications, \\ Xi'an 710061, Shaanxi, China \\ ${ }^{2}$ Shaanxi Coal Chemical Materials Storage and Transportation Co. LTD, Xi'an 710061, Shaanxi, China \\ ${ }^{3}$ Department of Statistics Faculty of Science, University of British Columbia, Vancouver, BC V6T 1R6, Canada \\ Correspondence should be addressed to Hongmei Shan; shmxiyou@163.com
}

Received 3 September 2021; Revised 5 January 2022; Accepted 6 January 2022; Published 30 January 2022

Academic Editor: Manuel De la Sen

Copyright (c) 2022 Hongmei Shan et al. This is an open access article distributed under the Creative Commons Attribution License, which permits unrestricted use, distribution, and reproduction in any medium, provided the original work is properly cited.

\begin{abstract}
Service quality is the soul of express enterprises forever. It is of great practical significance for winning customer satisfaction, improving the market competition, and realizing sustainable performance. Unlike tangible products, express delivery service has the characteristics of intangibility, heterogeneity, indivisibility, and instability. While customer demands are complex and changeable and unpredictable, incorporating complex customer requirements into service design has been a growing interest of researchers and practitioners. This paper proposed an optimization design framework based on the quantitative Kano (QKNO) and the fuzzy quality function deployment (FQFD) to effectively achieve the best matching of enterprise service elements under the uncertainty and imprecise judgment information. An empirical study is conducted to verify the feasibility of the proposed approach. The results show that the framework could guide the express company to prioritize the enterprise service elements to maximize customer satisfaction and provide a reasonable budget allocation scheme to set the best resources match. It has theoretical and practical meaning for express enterprises to implement customization service strategy and improve service quality under the limited budget, which could be further extended to other service industries to make optimization decisions.
\end{abstract}

\section{Introduction}

In the network economy era, the booming of online shopping blew out the prosperity of the express logistics industry (Subramanian et al.) [1]. Express delivery service has become a bridge and link between businesses and consumers. It is one of the essential competitive means for all enterprises to win higher customer's satisfaction (Dospinescu et al.) [2]. Especially in the particular period of the epidemic, the community delivery service of daily necessities is fast and convenient for the public to refrain from going outdoors as much as possible, such as going to markets or crowded restaurants. Compared with the product, express delivery service is perishable, changeable, indivisible, and intangible, while customer consumption behaviour is complex and changeable (Shan et al.) [3]. It is tricky for express service providers to continuously optimize the express service to effectively realize the best matching of customer demands and service elements in fuzzy language judgment information and increase the service quality level and enhance market competitiveness. It has significant theoretical and practical meaning to study how to accurately identify the relationship of customer satisfaction (CS) and different customer requirements and build a customerdriven service optimization design method for the express company.

Quality function deployment (QFD) is a customeroriented product/service innovation technique with extensive support in various fields of quality management and product/service innovation. It is a systematic method which effectively converts customer requirements (CRs, called customer demands, customer attributes, customer needs) to service elements (SEs, called designing characteristics, technical indicator) with operational processes of product/ 
service design in an orderly and reasonable manner (Vinodh et al.) [4]. Huo and Hong [5] pointed out that, in the design process of product service system, QFD can be used to realize the integration of customer requirements. It is mainly based on the combination of "customer voice" and product or service design and can be applied to improve service quality. For example, in service-oriented manufacturing, QFD can be applied to realize the design of product/service system based on customer experience. Fargnoli and Sakao [6] analysed the application of QFD in different fields based on case studies. Among them, in the product/service system (PSS) field, the service and product integration method based on QFD extends the traditional QFD. Haber and Fargnoli [7] proposed a new method to capture customer requirements based on the realization of the PSS function. It provides a practical answer to the demand for maximizing customer information by combining the quality function deployment for the product service systems (QFD for PSS) method with Thurstone's law of comparative judgments (LCJ). In the transformation process, correctly ranking the weight of CRs is a crucial and essential process because it subsequently has a significant impact on the target value of SEs setting. However, the procedure in QFD does not offer details on how to prioritize the CRs and get the ultimate results. Therefore, unremitting efforts must be made to correctly obtain the final importance rating of CRs/SEs to realize customer-oriented product development, especially in intangible express delivery service areas. A large number of relative literatures considered the importance rating of CRs. For example, Millen and Maggard [8] obtained the weight of SEs by analytic hierarchy process (AHP) and then determined the key optimization indicators of express service in the using of QFD. However, an expert's judgment (or perception) on the relevant weight of CRs is essentially inaccurate and uncertain, which reduces the objectivity and rationale of decision-making results. Zadeh [9], from the Department of Electrical Engineering of the University of California, Berkeley, set the Fuzzy Set Theory that has been widely applied in various fields to solve the fuzzy language evaluation information and uncertain issues. Khoo and Ho [10] proposed a fuzzy quality function deployment (Fuzzy QFD, FQFD) to address linguistic variables' ambiguity and multiplicity of meaning. Chan et al. [11] and Vanegas and Labib [12] rated the importance of CRs, which combines the fuzzy triangular numbers and entropy theory with QFD to consider the uncertain related with the mapping of customer's perception. Lan et al. [13] proposed a customer satisfaction evaluation model of manufacturing-driven logistics service based on Fuzzy Analytic Hierarchy Process (FAHP) and introduced the triangular fuzzy number into the FAHP method to determine the weight. Haber et al. [14] proposed translating CRs into PSS functionalities. Specifically, the quality function deployment for product service systems (QFD for PSS) method extends through the Kano model, which filters CRs effectively. In addition, based on the effectiveness of QFD for PSS, the fuzziness problem in PSS is reduced well through its integration with the FAHP method.

By the analysis above, the FQFD framework can determine the importance of CRs/SEs and achieve the transformation from CRs to SEs. However, customer preferences are varied and changeable, and the different types of CRs contribute to customer satisfaction (CS). In this case, it is not easy to ultimately reflect the relationship between CRs and CS, since meeting a certain CR does not represent a high level of customer satisfaction. Inspired by Fredrick Herzberg's Motivator-Hygiene Theory (Berger et al.) [15], the Kano model was proposed by Japanese quality management expert Kano et al. [16]. The Kano model believed that a two-dimensional model should be taken to acknowledge the quality of customer's subjective perceptions and objective performance of product/service, then to obtain the nonlinear relationship between customer satisfaction and product/service performance (Witell and Lo "fgren [17]; Chen and Su [18]). This model is undoubtedly of great value to grasp CRs and carry out scientific service decision-making. Based on this, Matzler et al. [19, 20] addressed the combination of the Kano model and QFD to make the product match customers' expectations. Hsuan and Chang [21] and Chun and Chuen [22] presented a robust design method associated with Kano's model with QFD to identify customer expectations and obtain the optimal combination of product design.

In summary, a few types of research on combinations of QFD and Kano models are used to research tangible product design. However, the procedure in QFD does not offer details on how to prioritize the CRs. There are some significant "research gaps" on the fuzziness of classification of CRs and inaccuracy identification of the relationship between CRs and CS in the process of QFD. Meanwhile, none of the studies provide robust guidance for customer-oriented product innovation and service optimization of express service enterprises to bring about higher customer satisfaction by integrating various functions. In the era of a competitive network economy, express companies have deeply realized the strategic significance of service optimization design guided by maximizing customer satisfaction, just like the case in other product/service industries. With this question in mind, some related descriptions tracked by this work team are provided in the following section. The literature review concludes that there is no framework for express company to effectively achieve the matching of CRs and SEs for maximizing the CS because of a lack of suitable methods for determining relative importance ratings of CRs and SEs in uncertainty and incomplete perceptions information.

In a different perspective, this paper aims to target the following issues. (1) In the optimum design of express service, how does the enterprise determine the priority of SEs to maximum the CS? And how does the enterprise allocate the budget reasonably under the limited constraints? (2) How to avoid the subjectivity of CRs' classification and establish the fitting relationship between CRs and CS accurately? (3) How to solve the randomness and ambiguity of experts' semantic evaluation in determining the importance of CRs? This study proposed an optimization decision framework for express service based on integrated quantitative Kano (QKANO) with a fuzzy QFD (FQFD) model to settle the above target problems. Compared with the 
previous researches, the merits of this study are as follows: (1) an optimization decision model of express service is built under the limited cost constraints; (2) the S-CR fitting functions based on the QKANO model is established to reflect the relationship of different type of CRs and CS; (3) the fuzzy triangle number is introduced into the QFD process to obtain the importance of CRs and to avoid the randomness and ambiguity of experts' semantic evaluation. This framework can effectively direct the express company to match the customer demands and the service elements and provide a powerful decision support approach for service planning and product optimization design.

The structure of this paper is as follows. The first section introduces the background. The second section provides a literature review. The third section proposes an optimum methodology for express delivery service, mainly including the evaluation method of CS based on QKANO, establishing of FQFD method for determining the importance of CRs/ SEs, and the construction of express service optimization decision-making model. The fourth section presents a case study to demonstrate the approach's feasibility. Lastly, management inspiration and research significance are remarked in the fifth section.

\section{Literature Review}

2.1. Kano Model and Its Application in Express Service. The Kano model is a classification and prioritization tool for customer needs, invented by Kano et al. [16]. It identifies and classifies the different customer needs by recognizing the nonlinear relationship between a product's performance and customer satisfaction, which provides the decision-making basis for the enterprise to improve the service quality. Then, it is widely used in various fields to analyse customer demand and manage the product/service quality. However, with only a few related articles in the express service realm, Huiskonen and Prittila [23] used the traditional Kano model to classify the logistics service elements and strategically planned for strengthening logistics service according to the classification results. Yoshimitsu et al. [24] combined the ideas of the Kano model with prospect theory and proposed a quantified evaluation method of customer satisfaction based on the service expectation information of users. Kim and Riew [25] put forward a quality improvement strategy for Korea's Express Mail Service (EMS) by using the traditional Kano and improvement Gap model to classify and prioritize the service quality attributes. To address the deficiencies of traditional Kano, Meng et al. [26] proposed a QKNO method to improve service quality in express service only by building the quantitative satisfaction index and importance index. Qingliang et al. [27] further adopted the Kano model to make a dynamic prediction of express service quality attributes at four time points in 2008, 2010, 2012, and 2013 in China based on GM $(1,1)$ and Markov Chain Model. This study provided testimony for the existence of substitution life cycles of service quality property. Li and Meng [28] also identified the key elements affecting customer satisfaction based on the moderated regression analysis to improve the defects of the traditional Kano model.
From the above analysis, it can be seen that only several papers discuss the improvement of express service quality with the traditional Kano method. Moreover, some scholars advanced the traditional Kano from different perspectives, such as prospect theory and the Gap model. However, these studies failed to define the fitting relationship between CRs attribute and CS value.

2.2. QFD and Its Application in Express Service. Quality function deployment (QFD), a perfect systematic design methodology of product/service driven by customer demand, was first put forward by Akao [29]. It maps the CRs to SEs and subsequently into parts characteristics, process plans, and production requirements by establishing a house of quality (HOQ), Figure 1.

In Figure 1, the importance of customer requirement (CR) is measured by the weight of each requirement. The correlation matrix of service element (SE) refers to the correlation strength among service elements. The relationship matrix (CR-SE) indicates the relationship between specific customer requirement and service element.

Stuart and Stephen [30] introduced QFD into service design since it came into being. Millen and Maggard [8] used QFD to realize the transformation of CRs into SEs of express enterprise resources, obtained service elements' weight by analytic hierarchy process (AHP), and then determined the key optimization indicators of express service. However, it ignores the subjectivity and arbitrariness of experts' judgment information when evaluating the importance of CRs and SEs, which will reduce the objectivity of decisionmaking results. For the uncertainty of experts' opinions in group decision-making, Xiao et al. [31] proposed a consensus-based FMEA method to deduce the orderly classification of potential fault modes and convey preferences. Based on the model-driven maximum consensus optimization and the minimum adjustment consensus model, both driven by social trust, Zhang et al. [32] proposed an interactive consensus reaching process to maximize the consensus level among decision-makers under assumptions. Zhang et al. [33] proposed an optimization model based on a consistency-driven method for confidence assessment of individual semantics in comparative language expression preference relationships (CLEPRs) to obtain a collective consensus solution.

To solve the inaccuracy and arbitrariness of evaluation information, Vanegas and Labib [12] studied the target values of relevant design parameters, established a Fuzzy QFD model, and showed the feasibility of a novel approach in car door design. Piyanee and Pisal [34] proposed a Fuzzy QFD approach for managing supply chain operations KPIs. Eleonora and Rizzi [35] extended the application of QFD in logistics services and established an FQFD model to obtain the optimal strategic actions by cost/benefit analysis. Considering the customer satisfaction and resources finitude, Wang et al. [36] studied a multiobjective integer programming model based on FQFD to determine the optimal logistics service portfolio. Lin and Pekkarinen [37] proposed a multilevel modular express service design including 


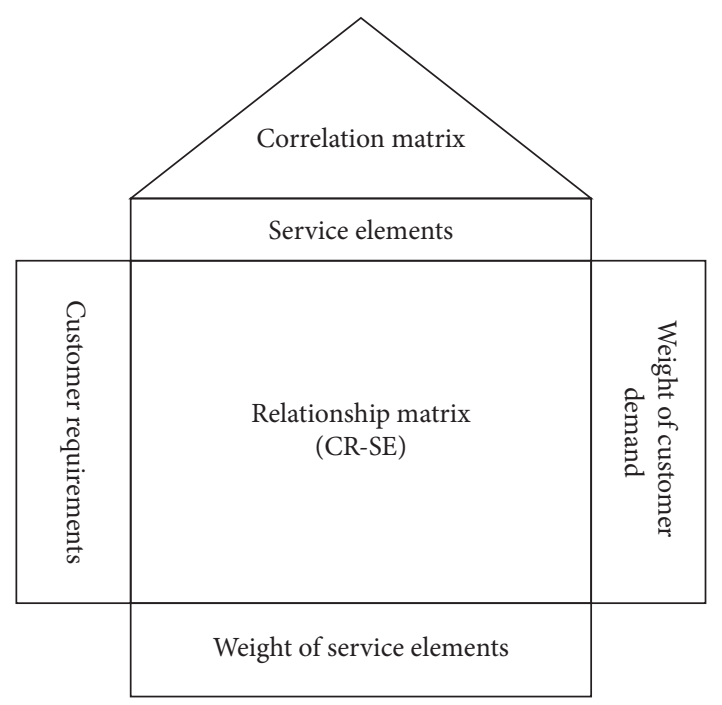

Figure 1: Basic composition of HOQ.

service, process, and activity modules based on HOQ and modularization theory. To identify the customer preferences and achieve higher customer satisfaction, Tan and Theresia [38] proposed an integrated model including SERVQUL, Kano, and QFD to assess customer satisfaction to guide the improvement of service quality attributes. Baki et al. [39] also used this combination approach for a well-known cargo company in Turkey, specifically defined the strengths and weaknesses of logistics services by SERVQUAL, categorized service quality attributes based on the Kano model, and then transformed the relevant result information into the QFD model. Nahm [40] studied a new framework to prioritize the CRs importance in the QFD process by combining the competitive benchmarking analysis with Kano's analysis. Furthermore, Nahm et al. [41] further improved the approach to prioritizing CRs in the QFD by developing the customer preference rating (CPR) and customer satisfaction rating (CSR) methods. The CSR method was integrated with Kano's model to capture the different impacts of CRs on customer satisfaction based on the competitive benchmarking analysis. Because of the typical imprecision or uncertainty of customer's judgment, Hsuan and Chang [21] further present a fuzzy nonlinear model to determine the performance level of each design requirement for maximizing customer satisfaction by the combination of the Kano model and QFD.

All in all, with the wide application and continuous promotion of QFD in services fields, the Kano model and fuzzy theory are, respectively, introduced to the crucial step of QFD in logistics service. However, none of the literature makes great efforts to solve the research gaps. One is the fuzziness of classification of CRs and inaccuracy identification of the relationship of CRs and CS in the traditional Kano model; the other is subjectivity and randomness of expert evaluation information in the application of QFD. Meanwhile, there is also no literature to provide robust guidance for customer-oriented product innovation and service optimization of express logistics enterprises to bring about higher customer satisfaction by integrating various functions.

\section{Methodology}

3.1. Research Framework. This study set up an optimal framework based on QKNO and FQFD to address these research gaps of express service making-decision. The method overcomes the imprecise and arbitrariness of the traditional Kano model in customer demand classification and inaccuracy identification of the relationship of CRs and CS. Moreover, it eliminates the subjectivity and ambiguity of expert evaluation information in the QFD process. The QKANO model is integrated into FQFD to realize the transformation from the weight of express service demand to the weight of express service elements and effectively make the optimization decision of express service. At the same time, it helps express enterprises maximize customer satisfaction under cost constraints.

The specific framework is shown in Figure 2. The first step is to establish the objective function of the optimization model, which includes the classification of CRs based on the QKANO method and the determination of fitting functions between CS and various CRs. This step could calculate the $\mathrm{CS}$ value by achievement of the individual category CR. The second step is the construction of FQFD to obtain the service elements (SEs) importance of express enterprises. It mainly realizes the transformation and matching of express CRs to SEs, including determining CRs importance, building a relationship matrix between CRs and express firm's SEs, and constructing the autocorrelation matrix of SEs. The results aim to establish constraints of the proposed optimization model. The third step is constructing an optimization model for express service based on the results of the above steps. Under budget and technical constraints, a mixed nonlinear programming model is established to maximize customer satisfaction. Finally, A practical case study is also conducted to demonstrate the feasibility of express service's proposed optimization approach.

\subsection{Construction of QKANO Model for Express Service}

3.2.1. Classification of Customer Requirements. In order to accurately meet the personalized customer demands, this study starts with establishing an express service optimization design group, whose members are composed of a project team member, enterprise manager, and frontline employees and experts from universities in different basins. According to the customer interview, the most concerned set of customer requirement $\mathrm{CR}=\left(\mathrm{CR}_{1}, \mathrm{CR}_{2}, \ldots \mathrm{CR}_{i}\right)$ is obtained after deliberations by design group; $\mathrm{CR}_{i}$ represents the $i$-th express service demand in CR, $i=(1,2, \cdots m)$. The customer survey is conducted to classify the various CR through a Kano questionnaire. The designed Kano questionnaire includes two forms of questions in positive and negative. The positive questions are the customers' service feeling when these demands are met, and the negative questions are the experience when the customer requirements are not satisfied. The level of service experiences from customer can be divided into five levels, including "Like," "Must-be," "Neutral," "Live with," 


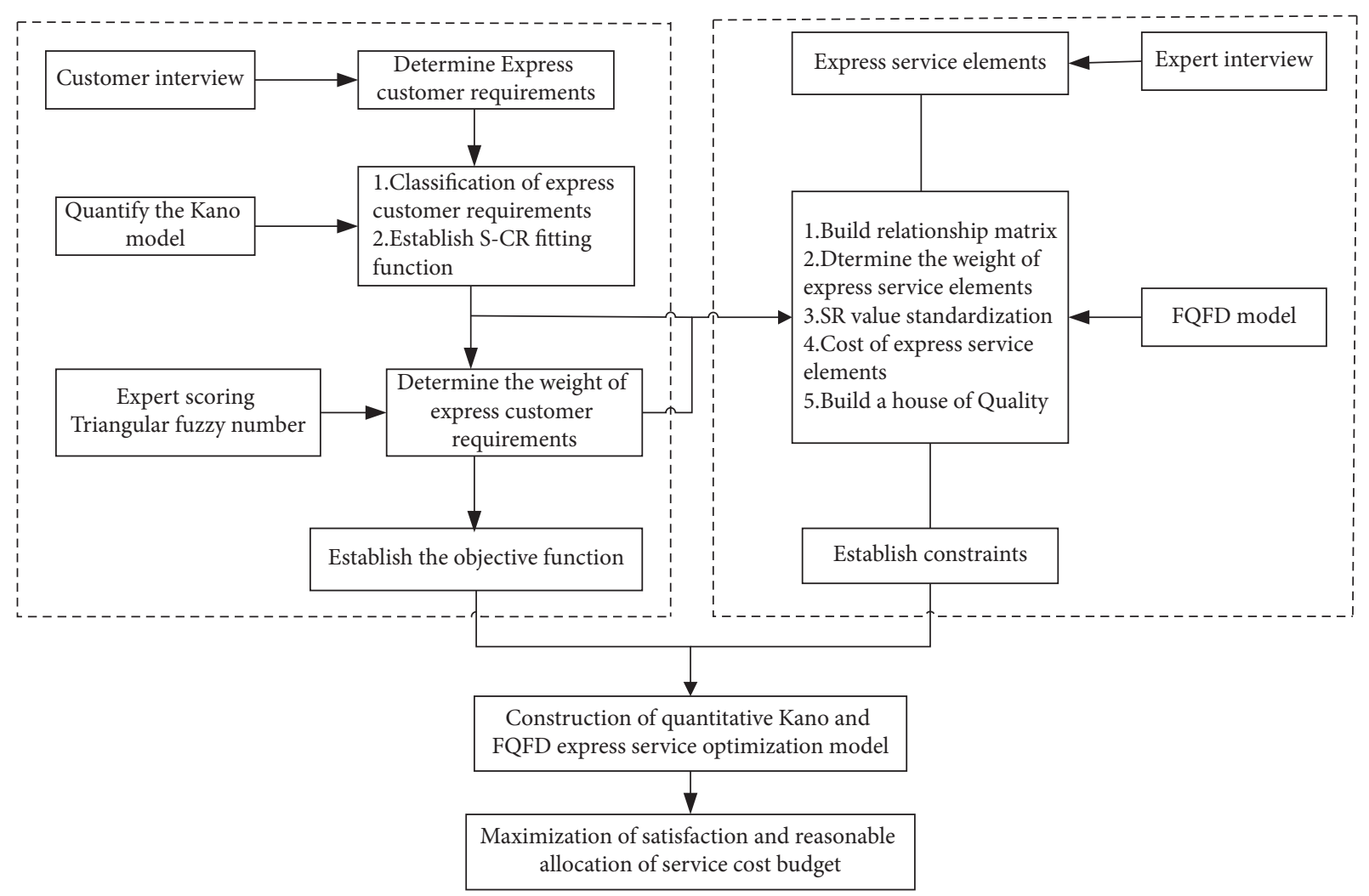

FIGURE 2: The framework of optimization design of express service based on QKANO and FQFD.

and "dislike." According to the positive and negative questions corresponding to each service element provided by the express company, the respondents will get 25 combinations. Each set of answers forms a classification table. The Kano classification assessment table is shown in Table 1, in which " $\mathrm{A}$ " represents attractive demand. "O" stands for one-dimension demand, " $M$ " represents the must-be type demand, "I" indicates indifference demand, " $R$ " is reverse demand, and " $Q$ " means the questionable demand.

After collecting questionnaires, SPSS23.0 and Excel2019 statistical software are used to process customer evaluation information. According to the classification criteria of the Kano model, the customer demand type with the highest frequency is selected as the final CR category.

\subsubsection{Construction of a Customer Satisfaction Fitting} Function. Berger [15] proposed the Better-Worse coefficient to measure the "price-performance ratio" of customer demands. Different customers have different expectations for service elements provided by enterprises. The values of customer satisfaction (CS) and customer dissatisfaction (DS) reflect the CS degree obtained by meeting or not meeting particular CRs. In this paper, the Better-Worse coefficient is calculated based on the selected frequency of CRs from the KANO questionnaire. The specific calculations are shown in formula (1):

Set $\mathrm{CS}_{i}$ as customer satisfaction for $\mathrm{CR}_{i}, \mathrm{DS}_{i}$ as customer dissatisfaction for $\mathrm{CR}_{i}$; then

$$
\begin{aligned}
\mathrm{CS}_{i} & =\frac{f_{A}+f_{O}}{f_{A}+f_{0}+f_{M}+f_{I}}, \\
\mathrm{DS}_{i} & =-\frac{f_{O}+f_{M}}{f_{A}+f_{0}+f_{M}+f_{I}} .
\end{aligned}
$$

In formula (1), $i$ indicates the order of service demand elements in the Kano questionnaire, $f_{A}$ represents the frequency of attractive demand, $f_{O}$ represents the frequency of one-dimension demand, $f_{M}$ represents the frequency of must-be demand, and $f_{I}$ represents the frequency of indifferent demand.

According to the study results of the Kano model by Fan and Zhao [42], for different types of CR, customer satisfaction curve trend is different, which means different types of functions $S_{i}=a f\left(y_{i}\right)+b$ to fit curves between CS and CRs (S-CR) for express delivery services. This paper establishes the fitting function curves of attractive demand, must-be demand, and one-dimension demand, as shown in Figure 3. The coordinate system is established with the Better-Worse coefficient (CS, DS) as the vertical axis and the achievement degree $\left(y_{i}\right)$ of customer demand as the horizontal axis. The CS point of a CR, $\left(1, \mathrm{CS}_{i}\right)$ in the $S$-CR curve of Figure 3, is the CS level when the CR is fully achieved. The DS point of a CR, $\left(0, \mathrm{DS}_{i}\right)$ on the coordinate axis, is the DS level when the CR is fully dissatisfied. Two characteristic points could be brought into the fitting function $\left(S_{i}=a f\left(y_{i}\right)+b\right)$ to be fitted by category. Then, the specific functional expressions between the realization degrees of 
TABLE 1: Kano evaluation table.

\begin{tabular}{lcccc}
\hline \multirow{2}{*}{ The requirement can achieve } & \multicolumn{3}{c}{ The requirement cannot achieve } \\
& Like & Must-be & Neutral & Live with \\
\hline Like & Q & A & D & A \\
Must-be & R & I & I & I \\
Neutral & R & I & I & I \\
Live with & R & I & R & I \\
Dislike & R & R & R & M \\
\hline
\end{tabular}

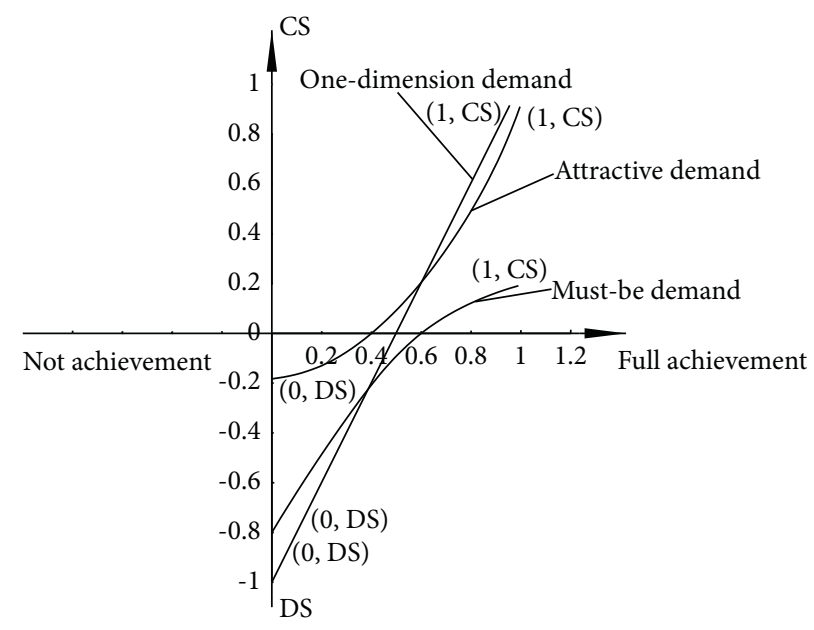

FIGURE 3: The function curve of customer satisfaction and CR achievement.

each CR category and CS degrees are obtained. $S_{i}$ represents $\mathrm{CS}$ for $\mathrm{CR}_{i}$, and $y_{i}$ represents the achievement degree of $\mathrm{CR}_{i}$; the range of $y_{i}$ is 0 to 1 . The curve parameters for different types of $\mathrm{CR}$ are notated as $a$ and $b$. The corresponding formulas are as follows from (2) to (4).

First, the fitting function of the one-dimension demand S-CR curve is $S=a_{1} y+b_{1}$, which is a linear function, and the curve parameters are $a_{1}$ and $b_{1}$ :

$$
a_{1}=\mathrm{CS}_{i}+\mathrm{DS}_{i}, b_{1}=\mathrm{DS}_{i} \text {. }
$$

Second, the fitting function of the attractive demand S-CR curve is $S=a_{2} e^{y}+b_{2}$, which is an exponential function, and the curve parameters are $a_{2}$ and $b_{2}$ :

$$
\begin{aligned}
& a_{2}=\frac{\left(\mathrm{CS}_{i}+\mathrm{DS}_{i}\right)}{(e-1)}, \\
& b_{2}=\frac{-\left(\mathrm{CS}_{i}-e \mathrm{DS}_{i}\right)}{(e-1)} .
\end{aligned}
$$

Third, the fitting function of the must-be demand S-CR curve is $S=-a_{3} e^{-y}+b_{3}$, which is also an exponential distribution function, and the curve parameters are $a_{3}$ and $b_{3}$ :

$$
\begin{aligned}
& a_{3}=\frac{e\left(\mathrm{CS}_{i}-\mathrm{DS}_{i}\right)}{(e-1)}, \\
& b_{3}=\frac{\left(e C S_{i}-\mathrm{DS}_{i}\right)}{(e-1)} .
\end{aligned}
$$

Based on the analysis above, three types of Kano S-CR fitting functions are as follows:

The fitting function of one-dimension $(O)$ demands is shown as follows:

$$
S_{i}=\left(\mathrm{CS}_{i}-\mathrm{DS}_{i}\right) y_{i}+\mathrm{DS}_{i}
$$

The fitting function of attractive $(A)$ demands is shown as follows:

$$
S_{i}=\frac{\left(\mathrm{CS}_{i}-\mathrm{DS}_{i}\right) e^{y_{i}}}{e-1}-\frac{\mathrm{CS}_{i}-e \mathrm{DS}_{i}}{e-1} .
$$
follows:

The fitting function of must-be $(M)$ demands is shown as

$$
S_{i}=\frac{e\left(\mathrm{CS}_{i}-\mathrm{DS}_{i}\right) e^{-y_{i}}}{e-1}-\frac{e C S_{i}-\mathrm{DS}_{i}}{e-1}
$$

3.3. Construction of FQFD Model for Express Service. The fuzzy theory supports decision-making processes based on imprecise and uncertain information. This theory emphasizes human thinking, reasoning, and cognition to things around. Its concepts are quite vague and inaccurate, so fuzzy logical concepts must be used to describe things in real life, to make up for the shortcomings of traditional sets that describe things by two-valued logic (Wang et al.) [43]. Zadeh, an expert in cybernetics in the United States, addressed the "membership function" to describe the 
intermediate transition of phenomenon differences, basically defining the fuzzy number as

$$
\widetilde{A}=\left\{\left|\left(x, u_{\tilde{A}}(x)\right)\right| x \in X\right\}, u_{\widetilde{A}}: X \longrightarrow[0,1], x \longrightarrow u_{\widetilde{A}}(x) .
$$

In formula (8), $X$ represents a universe set; $\widetilde{A}$ indicates a fuzzy set on $X$; and $\mathcal{u}_{\tilde{A}}(x)$ represents the membership degree of element $x$ to $\widetilde{A}^{A}$. $x$ equal to 1 indicates the element completely belongs to the set, and $x$ equal to 0 indicates the element is outside the range of fuzzy set. $u_{\tilde{A}}(x)$ is regarded as a set of triangular fuzzy numbers, denoted as $u_{\tilde{A}}(x)=(m, l, r)$. The function expression $\mathcal{u}_{\tilde{A}}(x)$ is shown in formula (9). The figure of triangular fuzzy number is shown in Figure 4, which indicates the membership functions of $x$ in different positions.

$$
\begin{aligned}
u_{\widetilde{A}}(x) & =(m, l, r)=\left(\max \left\{\frac{t-1}{T}, 0\right\}, \frac{t}{T}, \min \left\{\frac{t+1}{T}, 1\right\}\right), \\
t & =(0,1 \cdots T) .
\end{aligned}
$$

In this section, the triangle fuzzy number is introduced to obtain the importance of key decision elements accurately. The steps of designed FQFD method are specified as follows:

(1) Determine the set of customer demand (CR) set and its weight $(W)$. A service design team are set up, and a set of demands $\mathrm{CR}=\left(\mathrm{CR}_{1}, \mathrm{CR}_{2}, \ldots \mathrm{CR}_{i}\right)$ is determined on the basis of customer interviews, where $\mathrm{CR}_{i}$ represents the $i$-th express service demand, $i=(1,2, \cdots m)$. The expert $E_{k}(k=1,2, p)$ evaluates the importance of customer demands within five-tier scale semantic evaluation variables, which are very unimportant (VL), unimportant (L), medium (M),

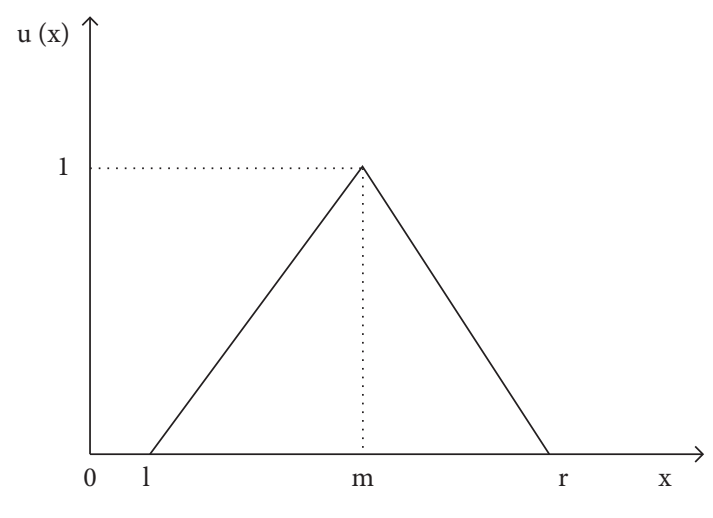

Figure 4: Triangular ambiguity.

important $(\mathrm{H})$, and very important $(\mathrm{VH})$. According to the triangular fuzzy formula (9), when $T=4$, the triangular fuzzy comments corresponding to each semantics scale level will be $\mathrm{VL}=(0,0,0.25), L=(0$, $0.25,0.5), M=(0.25,0.5,0.75), \mathrm{VH}=(0.5,0.75,1)$, and $\mathrm{VH}=(0.75,1,1)$. The experts' weight vectors are indicated as $W E=\left(W E_{1}, W E_{2}, \cdots W E_{p}\right)$, where $W E_{k}$ represents the weight value given by $k$-th expert, and $0 \leq W E_{k} \leq 1, \sum_{k=1}^{o} W E_{k}=1$. Suppose the fuzzy matrix of the evaluation of expert $E$ to customer demands is $\widetilde{A}=\left[\widetilde{a}_{k i}\right]_{p \times m}$, where $\tilde{a}_{k i}=\left(\tilde{a}_{k i}^{1}, \tilde{a}_{k i}^{2}, \tilde{a}_{k i}^{3}\right) ; \quad \tilde{a}_{k i}$ represents the semantics evaluation information of the $k$-th expert to the number of $i$ demands. Therefore, the fuzzy weight vector of customer demands $\widetilde{w}_{i}=\left(\widetilde{w}_{1}, \widetilde{w}_{2}, \cdots \widetilde{w}_{m}\right)$ is produced by the combination of weight value by experts, where $\widetilde{w}_{i}=\left(\widetilde{w}_{i}^{1}, \widetilde{w}_{i}^{2}, \widetilde{w}_{i}^{3}\right)$, and formula (10) is as follows:

$$
\widetilde{w}_{i}=\left(w_{i}^{1}, w_{i}^{2}, w_{i}^{3}\right)=\left(\sum_{k=1}^{o} W E_{k} w_{k i}^{1}, \sum_{k=1}^{o} W E_{k} w_{k i}^{2}, \sum_{k=1}^{o} W E_{k} w_{k i}^{3}\right), i=(1,2, \cdots m) .
$$

To obtain the relative weight of CRs $w_{i}^{\prime}$, $w_{i}^{\prime}=\left(w_{1}, w_{2} \cdots w_{m}\right)$, the inverse fuzzy algorithm of fuzzy number is used to defuzzify the fuzzy weight of customer demand. Formula (11) is as follows:

$$
w_{i}^{\prime}=\frac{\left(w_{i}^{1}+4 w_{i}^{2}+w_{i}^{3}\right)}{6} .
$$

The absolute weight of CR is calculated by normalizing the weight of customer demand, shown as follows:

$$
w_{i}=\frac{w_{i}^{\prime}}{\sum_{i=1}^{m} w_{i}^{\prime}}
$$

(2) Determine the set of express service element (SE). The full set $\mathrm{SE}=\left(\mathrm{SE}_{1}, \mathrm{SE}_{2}, \ldots \mathrm{SE}_{j}\right)$, which is associated with all $C R_{s}$, is obtained by the investigation of service design team in actual enterprise, where $S E_{j}$ represents the $\mathrm{j}$-th express service element, $j=(1,2, \cdots n)$.

(3) Determine the relationship matrix between CRs and express SEs. Based on the design ideas from QFD, the service design team takes a three-level semantic scale to describe the relationship between CRs and SEs, namely, weak $(\square)$, medium $(\diamond)$, and strong $(\diamond)$. According to formula (9), let $T=2$; the triangle fuzzy comments corresponding to each semantic scale level are $\square=(0,0,0.5), \diamond=(0,0.5,1)$, and $\diamond=(0.5,1$, $1)$. The fuzzy matrix of relation between CRs and express SEs is $\widetilde{R}=\left[\widetilde{r}_{i j}\right]_{m \times n}$, where $r_{i j}$ represents the correlational degree between the customer requirement $\left(C R_{i}\right)$ and service elements $\left(S E_{j}\right)$. To simplify the calculation, the matrix $\widetilde{R}=\left[\widetilde{r}_{i j}\right]_{m \times n}$ is deblurred to obtain the de-fuzzified matrix $R=\left[r_{i j}\right]_{m \times n}$. 
(4) Determine the autocorrelation matrix $\tilde{Y}$ of express SEs. Combine the self-correlation semantic information of the express delivery SEs, that is, very low (VL), low (L), medium (M), high $(\mathrm{H})$, and very high (VH). According to formula (9), let $T=4$; the triangular fuzzy comments corresponding to each medium scale level are $\mathrm{VL}=(0,0,0.25), L=(0,0.25$, $0.5), M=(0.25,0.5,0.75), \mathrm{VH}=(0.5,0.75,1)$, and $\mathrm{VH}=(0.75,1,1)$. The autocorrelation fuzzy matrix of relation of SEs is $\tilde{Y}=\left[\tilde{y}_{j k}\right]_{n \times n}$, where $y_{j k}$ represents the correlational degree between the $\mathrm{SE}_{j}$ and the $\mathrm{SE}_{k}$. To simplify the calculation, the matrix $\widetilde{Y}=\left[\tilde{y}_{j k}\right]_{n \times n}$ is deblurred to obtain the deblurred matrix $Y=\left[y_{j k}\right]_{n \times n}$.

(5) Determine the weight $(V)$ of express delivery SEs. In this step of QFD model, the weight of express SEs is determined by the weight of CRs $(w)$, the relationship matrix of customer demand, express service elements $R=\left[r_{i j}\right]_{m \times n}$, and the autocorrelation matrix of express SEs $Y=\left[y_{j k}\right]_{n \times n}$. The relationship matrix between CRs and SEs in the house of quality (HOQ) is standardized to combine with the autocorrelation matrix of the express SEs (Chen and Weng) [44]. The standardization formula is as follows:

$$
R_{i j}^{n o r m}=\frac{\sum_{k=1}^{n} R_{i k} \times Y_{k j}}{\sum_{j=1}^{n} \sum_{k=1}^{n} R_{i j} \times Y_{j k}}, i=1,2, \cdots m ; j=1,2, \cdots n .
$$

If the importance vector of express service CRs is $w_{i}=\left(w_{1}, w_{2} \cdots w_{m}\right)$, then the weight of express SEs is $v_{i}=\left(v_{1}, v_{2}, \cdots v_{k}\right)$, where $\sum_{i=1}^{n} v_{i}=1$. The weight of express service element formula is as follows:

$$
v_{i}=\sum_{i=1}^{n} w_{i} * r_{i j}
$$

3.4. Construction of Optimization Decision Model Based on the QKNO and FQFD. The objective of the optimization decision model is to obtain maximum CS under limited resources. The S-CR relationship functions between CRs and CS levels are established based on the above analysis. The CS of each CR $\left(s_{i}\right)$ can be calculated by formula (16). The total CS is recorded as $S\left(y_{1}, y_{2} \ldots y_{m}\right)$, representing the weighted sum of CS of each CR.

$$
S\left(y_{1}, y_{2} \cdots y_{m}\right)=\sum_{i=1}^{m} w_{i} s_{i}
$$

In formula (15), $w_{i}$ is the weighted vector of each $\mathrm{CR}$, $w_{i}=\left(w_{1}, w_{2} \cdots w_{m}\right)^{T}, 0<w_{i}<1$, and

$$
s_{i}=\left\{\begin{array}{l}
S_{i}=\left(\mathrm{CS}_{i}-\mathrm{DS}_{i}\right) y_{i}+\mathrm{DS}_{i} \\
S_{i}=\frac{\left(\mathrm{CS}_{i}-\mathrm{DS}_{i}\right) e^{y_{i}}}{e-1}-\frac{\mathrm{CS}_{i}-e \mathrm{DS}_{i}}{e-1} \\
S_{i}=\frac{e\left(\mathrm{CS}_{i}-\mathrm{DS}_{i}\right) e^{-y_{i}}}{e-1}-\frac{e \mathrm{CS}_{i}-\mathrm{DS}_{i}}{e-1} \\
w_{1}+w_{2}+\cdots+w_{m}=1 .
\end{array}\right.
$$

The constraint of the optimization decision model is the budget funds of SEs to be optimized. The cost index $\left(b_{j}\right)$ of each SE can be obtained from the actual investigation of express enterprises. $b_{j}$ is the cost for express enterprise to improve a certain SE to meet CRs. Let the total cost of SEs be $B$. Since all SEs are discrete, it is necessary to add a binary random variable $x_{j k}$ (Ji et al. [45]). The value of $x_{j k}$ is either 0 or 1 . It is recorded as 1 when the service element $\left(\mathrm{SE}_{j}\right)$ is selected to meet the customer requirement $\left(\mathrm{CR}_{i}\right)$ and 0 when not selected. For each service element $\left(\mathrm{SE}_{j}\right)$, there is a set of selectable discrete values obtained by the brainstorming of the expert team. Each set has a satisfaction level $d_{k j}$. Therefore, $x_{j}$ is equal to the fulfilment level of the selected $\mathrm{SE}_{j}$ value. Let $x_{j}$ be the fulfilment level of express service elements, as follows:

$$
x_{j}=\sum_{k=1}^{p} x_{j k} d_{k j}
$$

Then, the discrete SE values are mapped to customer requirement achievement levels to establish the mathematical programming model. The optimization decision model of express service is built by the combination of the objective function and its constraints as follows:

$$
\operatorname{MAX} \sum_{i=1}^{m} w_{i} s_{i} \text {. }
$$

$$
s_{i}=\left\{\begin{array}{l}
S_{i}=\left(\mathrm{CS}_{i}-\mathrm{DS}_{i}\right) y_{i}+\mathrm{DS}_{i} \\
S_{i}=\frac{\left(\mathrm{CS}_{i}-\mathrm{DS}_{i}\right) e^{y_{i}}}{e-1}-\frac{\mathrm{CS}_{i}-e \mathrm{DS}_{i}}{e-1} \\
S_{i}=\frac{e\left(\mathrm{CS}_{i}-\mathrm{DS}_{i}\right) e^{-y_{i}}}{e-1}-\frac{e \mathrm{CS}_{i}-\mathrm{DS}_{i}}{e-1} \\
w_{1}+w_{2}+\cdots+w_{m}=1 .
\end{array}\right.
$$

This is subject to 


$$
\begin{aligned}
x_{j} & =\sum_{k=1}^{p} x_{j k} d_{k j} . \\
\sum_{k=1}^{p} x_{j k} & =1 . \\
\sum_{j=1}^{n} b_{j} x_{j} & \leq B . \\
y_{i} & =\sum_{j=1}^{n} R_{i j} x_{j}, i=1,2, \cdots m . \\
0 & \leq x_{j} \leq 1, j=1,2, \cdots n . \\
x_{j k} & \in(0,1) .
\end{aligned}
$$

In the above optimization modelling, formula (18) indicates that service optimal design aims to maximize customer satisfaction. Formula (20) illustrates the standardization of the discrete SE; formula (21) represents the constraint that only one service element can be selected for discrete SE value. Formula (22) shows that the total cost of the optimal design cannot exceed the budget; formula (23) illustrates that the $S E$ fulfillment level is converted into the achievement level of CR; formula (24) indicates that the range of $x_{j}$ is 0 to 1 , and formula (25) indicates that $x_{j k}$ is a binary variable. The optimized design model of express delivery service was established as a nonlinear programming model, which can be solved by using Lingo 16.0 software. The final decision scheme of express service elements can be determined.

\section{Case Study}

In order to verify the feasibility of proposed optimization model, a branch of SF express in Xi'an is selected as the case study sample. It is convenient to investigate and acquire data as our school-enterprise cooperation unit. Xi'an SF is a third-party logistics enterprise serving medium and high customers. The company has always adhered to the purpose of "Service First, Customer First," constantly improving service and product structure. In order to better meet the customer demands and increase the CS, the company's budget investment is 1.4 million yuan to optimize the SEs.

Firstly, the express service optimization design group is set up, including $2 \mathrm{Xi}$ 'an SF department managers, 1 SF frontline courier, and 3 experts from the relevant research institutions. The research team collects customer requirements information through customer interviews. After merging and deleting some duplicate or unimportant items, 16 CRs are identified to stand for the biggest concerns of customers, including "accurately fulfilling the service commitment" $\left(\mathrm{CR}_{1}\right)$, "7-day free charge kept for undelivered items" $\left(\mathrm{CR}_{2}\right)$, "insured services" $\left(\mathrm{CR}_{3}\right)$, "packaging service available" $\left(\mathrm{CR}_{4}\right)$, "after-sales service guarantee" $\left(\mathrm{CR}_{5}\right)$, "rapid response and handling orders" $\left(\mathrm{CR}_{6}\right)$, "timely update logistics tracking information" $\left(\mathrm{CR}_{7}\right)$, "prompt response to return and replacement" (CR8), "notification to sender and consignee by Short Messaging Service (SMS)" ( $\left.\mathrm{CR}_{9}\right)$, "clarity of tracking number" $\left(\mathrm{CR}_{10}\right)$, "reliable service staff” $\left(\mathrm{CR}_{11}\right)$, "professional personnel" $\left(\mathrm{CR}_{12}\right)$, "return visit to key customers" $\left(\mathrm{CR}_{13}\right)$, "available delivery time" $\left(\mathrm{CR}_{14}\right)$, “appointment for pickup service" $\left(\mathrm{CR}_{15}\right)$, and "authorizable pickup" $\left(\mathrm{CR}_{16}\right)$.

Using the designed Kano questionnaire, a customer demand survey is performed from December 2019 to March 2020 to classify express CRs. The respondents are selected randomly from the customer of Xi'an SF. A total of 400 questionnaires are sent out in online and face-to-face form; 380 of them are collected. After eliminating the invalid questionnaires, a total of 296 valid questionnaires were collected, which covered a $77.9 \%$ effective rate. The survey objects are mainly students, engineers, managers, teachers, and other employees. $97.29 \%$ of responders have purchased Xi' an SF express delivery, and 32.6\% received 50-100 items from Xi'an SF per year. In this paper, SPSS23.0 software is used to test the reliability and validity of data. The reliability test results show that Cronbach's values of the positive and negative problems are 0.940 and 0.914 , which means the questionnaire is reliable. The validity test results show that the KMO measure values were 0.921, indicating the questionnaire is trustable for factor analysis. Bartlett's test of sphericity is less than 0.001 , reaching a significant probability. Therefore, it can be concluded that the data of the questionnaire has good validity. SPSS 23.0 and Excel 2019 are used to conduct statistics according to the Kano method, and 16 different CR classification results are obtained (see Table 2). The customer satisfaction (CSi) and customer dissatisfaction (DSi) are calculated by using the formula (1) in section 3.2.2. The results are shown in Table 2.

According to the Kano classification results in Table 2 and formulas (2-4), the S-CR fitting function between each CR and CS can be determined in Table 3.

The weight of CRs is described by the expert scoring method. Four experts are selected in this paper. The expert set and the corresponding weights are shown in Table 4.

The experts evaluated the importance of CR according to 5 granularity comments. The fuzzy weight of express $\widetilde{w}_{i}$ delivery customer demand can be obtained through formula (10). After defuzzification with formula (11), the final CRs weight $w_{i}$ can be obtained by combining formula (12). The results of expert evaluation information and CRs weight are shown in Table 5.

Soon afterwards, the express service optimization design team investigated the express SEs provided by Xi'an SF. After deliberate analysis and deletion of some irrelevant items, 9 express SEs were determined, including standardization of operation process $\left(\mathrm{SE}_{1}\right)$, smooth customer communication channel $\left(\mathrm{SE}_{2}\right)$, personalized service $\left(\mathrm{SE}_{3}\right)$, delivery network coverage $\left(\mathrm{SE}_{4}\right)$, customer knowledge management $\left(\mathrm{SE}_{5}\right)$, timely update of delivery information $\left(\mathrm{SE}_{6}\right)$, after-sales service management $\left(\mathrm{SE}_{7}\right)$, staff training management $\left(\mathrm{SE}_{8}\right)$, and perfect complaint mechanism $\left(\mathrm{SE}_{9}\right)$.

Subsequently, the relationship matrix between CRs and express SEs is established according to the scale of the three- 
Table 2: Kano classification of customer requirement and the Better-Worse coefficient.

\begin{tabular}{|c|c|c|c|c|c|c|c|c|c|}
\hline Customer requirements & $\mathrm{A}$ & $\mathrm{M}$ & $\mathrm{O}$ & $\mathrm{I}$ & Q & $\mathrm{R}$ & Categories & $\mathrm{CS}_{i}$ & $\mathrm{DS}_{i}$ \\
\hline $\mathrm{CR}_{1}$ & 40 & 124 & 86 & 33 & 10 & 3 & $\mathrm{M}$ & 0.4300 & -0.7167 \\
\hline $\mathrm{CR}_{2}$ & 78 & 44 & 103 & 54 & 9 & 8 & $\mathrm{O}$ & 0.6285 & -0.5104 \\
\hline $\mathrm{CR}_{3}$ & 39 & 116 & 88 & 45 & 7 & 1 & M & 0.4305 & -0.6915 \\
\hline $\mathrm{CR}_{4}$ & 74 & 31 & 115 & 43 & 12 & 21 & $\mathrm{O}$ & 0.6873 & -0.5310 \\
\hline $\mathrm{CR}_{5}$ & 39 & 118 & 88 & 43 & 7 & 1 & M & 0.4050 & -0.6983 \\
\hline $\mathrm{CR}_{6}$ & 81 & 31 & 116 & 52 & 6 & 10 & $\mathrm{O}$ & 0.6888 & -0.5140 \\
\hline $\mathrm{CR}_{7}$ & 37 & 111 & 59 & 73 & 8 & 8 & M & 0.3333 & -0.5903 \\
\hline $\mathrm{CR}_{8}$ & 52 & 49 & 158 & 29 & 8 & 0 & $\mathrm{O}$ & 0.7095 & -0.6993 \\
\hline $\mathrm{CR}_{9}$ & 154 & 15 & 58 & 59 & 8 & 2 & A & 0.7211 & -0.2483 \\
\hline $\mathrm{CR}_{10}$ & 35 & 111 & 68 & 69 & 8 & 5 & M & 0.3540 & -0.6151 \\
\hline $\mathrm{CR}_{11}$ & 50 & 42 & 129 & 56 & 10 & 9 & $\mathrm{O}$ & 0.6240 & -0.5958 \\
\hline $\mathrm{CR}_{12}$ & 59 & 49 & 122 & 51 & 7 & 8 & $\mathrm{O}$ & 0.6285 & -0.5938 \\
\hline $\mathrm{CR}_{13}$ & 135 & 13 & 63 & 69 & 6 & 10 & A & 0.6923 & -0.2657 \\
\hline $\mathrm{CR}_{14}$ & 107 & 48 & 71 & 63 & 5 & 2 & A & 0.6054 & -0.4048 \\
\hline $\mathrm{CR}_{15}$ & 128 & 25 & 91 & 44 & 5 & 3 & A & 0.7474 & -0.3959 \\
\hline $\mathrm{CR}_{16}$ & 126 & 34 & 82 & 48 & 4 & 2 & A & 0.7075 & -0.3946 \\
\hline
\end{tabular}

Note: "A" represents attractive requirement, "O" represents one-dimension requirement, "M" represents the must-be requirement, "I" represents indifferent requirement, " $R$ " represents reverse requirement, and " $Q$ " represents Questionable requirement.

TABLE 3: S-CR relationship fitting function.

\begin{tabular}{|c|c|c|c|c|c|}
\hline Customer requirements & Categories & $a$ & $b$ & $f\left(y_{i}\right)$ & $S_{i}=a f\left(y_{i}\right)+b$ \\
\hline$\overline{\mathrm{CR}_{1}}$ & $\mathrm{M}$ & 1.8141 & 1.0974 & $-e^{-y_{1}}$ & $S_{1}=-1.8141 e^{-y_{1}}+1.0974$ \\
\hline $\mathrm{CR}_{2}$ & $\mathrm{O}$ & 1.1389 & -0.5104 & $y_{2}$ & $S_{2}=1.1389 y_{2}-0.5104$ \\
\hline $\mathrm{CR}_{3}$ & M & 1.7750 & 1.0835 & $-e^{-y_{2}}$ & $S_{3}=-1.7750 e^{-y_{3}}+1.0835$ \\
\hline $\mathrm{CR}_{4}$ & $\mathrm{O}$ & 1.2183 & -0.5310 & $y_{4}$ & $S_{4}=1.2183 y_{4}-0.5310$ \\
\hline $\mathrm{CR}_{5}$ & M & 1.7454 & 1.0471 & $-e^{-y_{5}}$ & $S_{5}=-1.7454 e^{-y_{5}}+1.0471$ \\
\hline $\mathrm{CR}_{6}$ & $\mathrm{O}$ & 1.2028 & -0.514 & $y_{6}$ & $S_{6}=1.2028 y_{6}-0.5140$ \\
\hline $\mathrm{CR}_{7}$ & M & 1.4611 & 0.8708 & $-e^{-y_{7}}$ & $S_{7}=-1.4611 e^{-y_{7}}+0.8708$ \\
\hline $\mathrm{CR}_{8}$ & $\mathrm{O}$ & 1.4088 & -0.6993 & $y_{8}$ & $S_{8}=1.4088 y_{8}-0.6993$ \\
\hline $\mathrm{CR}_{9}$ & A & 0.5642 & -0.8125 & $e^{y_{9}}$ & $S_{9}=0.5642 e^{-y_{9}}-0.8125$ \\
\hline $\mathrm{CR}_{10}$ & M & 1.5331 & 0.9180 & $-e^{-y_{10}}$ & $S_{10}=-1.5331 e^{-y_{10}}+0.9180$ \\
\hline $\mathrm{CR}_{11}$ & $\mathrm{O}$ & 1.2198 & -0.5958 & $y_{11}$ & $S_{11}=1.2198 y_{11}-0.5958$ \\
\hline $\mathrm{CR}_{12}$ & $\mathrm{O}$ & 1.2223 & -0.5938 & $y_{12}$ & $S_{12}=1.2223 y_{12}-0.5938$ \\
\hline $\mathrm{CR}_{13}$ & $\mathrm{~A}$ & 0.5575 & -0.8232 & $e^{y_{13}}$ & $S_{13}=0.5575 e^{-y_{13}}-0.8232$ \\
\hline $\mathrm{CR}_{14}$ & A & 0.6654 & -0.9927 & $e^{y_{14}}$ & $S_{14}=0.6654 e^{-y_{14}}-0.9927$ \\
\hline $\mathrm{CR}_{15}$ & $\mathrm{~A}$ & 0.6654 & -1.0613 & $e^{y_{15}}$ & $S_{15}=0.6654 e^{-y_{15}}-1.0613$ \\
\hline $\mathrm{CR}_{16}$ & A & 0.6414 & -1.0360 & $e^{y_{16}}$ & $S_{16}=0.6414 e^{-y_{16}}-1.0360$ \\
\hline
\end{tabular}

TABLE 4: Expert information set.

\begin{tabular}{lcc}
\hline Experts & Character description & $W_{k}$ \\
\hline$E_{1}$ & Marketing department manager & 0.3 \\
$E_{2}$ & Product department manager & 0.2 \\
$E_{3}$ & Logistics committee expert & 0.3 \\
$E_{4}$ & University logistics professor & 0.2 \\
\hline
\end{tabular}

level evaluation set, as shown in Table 6. Meanwhile, the autocorrelation matrix of express SEs is established according to the scale of the five-level evaluation set, as shown in Table 7.

According to Tables 6 and 7, the relationship matrix between CRs and express SEs is obtained $\widetilde{R}=\left[\widetilde{r}_{i j}\right]_{m \times n}$ as well as SEs autocorrelation matrix $\widetilde{Y}=\left[\widetilde{y}_{j j}\right]_{n \times n}$. The matrix after defuzzification is $R=\left[r_{i j}\right]_{m \times n}$ and $Y=\left[y_{j j}\right]_{n \times n}$. According to formula (13), the standardized matrix results between CRs and express SEs are calculated, as shown in Table 8. The weight of express SEs is obtained by formula (14). The HOQ is established based on the calculation results, including weights of customer demand $w$, the standardized matrix results $R^{\text {norm }}$ between customer demand and express service elements, the weights of express service elements $V$, and the budget costs $b$, as shown in Table 8. Among them, the optimized cost of each SE in Table 8 is provided based on the comprehensive consideration of the disposable investment of the enterprise and the net present value of operating costs in the recent three years.

In the case study, since express enterprises provide 9 discrete SEs to meet 16 various CRs, a binary variable $x_{j k}$ is added to reflect the corresponding relationship between SEs and CRs. The value of $x_{j k}$ is 0 or 1 . It is marked as 1 when $\mathrm{SE}_{j}$ is selected to meet the $\mathrm{CR}_{i}$; otherwise, it is marked as 0 . This paper followed the random coefficients' binary choice model by Eric Gautier [46] in processing the discrete random variables. The expert group 
TABLE 5: CR importance evaluation information and its weight.

\begin{tabular}{|c|c|c|c|c|c|c|}
\hline Customer requirements & $E_{1} w_{1}=0.3$ & $E_{2} w_{2}=0.2$ & $\mathrm{E} 3 w_{3}=0.3$ & $E_{4} w_{4}=0.2$ & CR fuzzy weighted $\widetilde{w}_{i}$ & Final CR weight $w_{i}$ \\
\hline $\mathrm{CR}_{1}$ & $\mathrm{VH}$ & $\mathrm{M}$ & $\mathrm{VH}$ & $\mathrm{M}$ & $(0.55,0.8,0.9)$ & 0.0685 \\
\hline $\mathrm{CR}_{2}$ & M & $\mathrm{H}$ & $\mathrm{VH}$ & M & $(0.45,0.7,0.875)$ & 0.0608 \\
\hline $\mathrm{CR}_{3}$ & $\mathrm{M}$ & $\mathrm{L}$ & $\mathrm{M}$ & $\mathrm{H}$ & $(0.25,0.5,0.75)$ & 0.0442 \\
\hline $\mathrm{CR}_{4}$ & $\mathrm{H}$ & $\mathrm{H}$ & $\mathrm{VH}$ & $\mathrm{VH}$ & $(0.625,0.875,1)$ & 0.0755 \\
\hline $\mathrm{CR}_{5}$ & $\mathrm{VH}$ & $\mathrm{H}$ & $\mathrm{M}$ & $\mathrm{VH}$ & $(0.55,0.8,0.925)$ & 0.0689 \\
\hline $\mathrm{CR}_{6}$ & M & $\mathrm{H}$ & $\mathrm{H}$ & M & $(0.525,0.775,0.95)$ & 0.0674 \\
\hline $\mathrm{CR}_{7}$ & $\mathrm{VH}$ & $\mathrm{H}$ & $\mathrm{L}$ & $\mathrm{VH}$ & $(0.475,0.725,0.85)$ & 0.0622 \\
\hline $\mathrm{CR}_{8}$ & $\mathrm{VH}$ & $\mathrm{H}$ & $\mathrm{H}$ & $\mathrm{VH}$ & $(0.625,0.875,1)$ & 0.0755 \\
\hline $\mathrm{CR}_{9}$ & M & $\mathrm{H}$ & $\mathrm{L}$ & M & $(0.225,0.475,0.725)$ & 0.0420 \\
\hline $\mathrm{CR}_{10}$ & $\mathrm{VH}$ & $\mathrm{H}$ & M & $\mathrm{VH}$ & $(0.55,0.8,0.925)$ & 0.0689 \\
\hline $\mathrm{CR}_{11}$ & M & $\mathrm{M}$ & $\mathrm{H}$ & $\mathrm{L}$ & $(0.275,0.525,0.775)$ & 0.0464 \\
\hline $\mathrm{CR}_{12}$ & $\mathrm{H}$ & M & $\mathrm{VH}$ & M & $(0.475,0.725,0.9)$ & 0.0630 \\
\hline $\mathrm{CR}_{13}$ & $\mathrm{VH}$ & $\mathrm{VH}$ & $\mathrm{H}$ & $\mathrm{H}$ & $(0.625,0.875,1)$ & 0.0755 \\
\hline $\mathrm{CR}_{14}$ & $\mathrm{M}$ & $\mathrm{H}$ & $\mathrm{VH}$ & $\mathrm{VH}$ & $(0.55,0.8,0.925)$ & 0.0689 \\
\hline $\mathrm{CR}_{15}$ & $\mathrm{H}$ & $\mathrm{H}$ & M & M & $(0.375,0.625,0.875)$ & 0.0552 \\
\hline $\mathrm{CR}_{16}$ & $\mathrm{VH}$ & M & $\mathrm{H}$ & M & $(0.4,0.65,0.9)$ & 0.0574 \\
\hline
\end{tabular}

Notes: VL: very unimportant; L: unimportant; M: medium; H: important; VH: very important.

TABle 6: Relationship matrix between CRs and SEs.

\begin{tabular}{|c|c|c|c|c|c|c|c|c|c|}
\hline & $\mathrm{SE}_{1}$ & $\mathrm{SE}_{2}$ & $\mathrm{SE}_{3}$ & $\mathrm{SE}_{4}$ & $\mathrm{SE}_{5}$ & $\mathrm{SE}_{6}$ & $\mathrm{SE}_{7}$ & $\mathrm{SE}_{8}$ & $\mathrm{SE}_{9}$ \\
\hline $\mathrm{CR}_{1}$ & $\diamond$ & $\diamond$ & $\diamond$ & $\square$ & $\diamond$ & $\diamond$ & $\diamond$ & $\square$ & $\diamond$ \\
\hline $\mathrm{CR}_{2}$ & $\square$ & $\diamond$ & $\bullet$ & $\square$ & $\square$ & $\square$ & $\square$ & $\square$ & $\square$ \\
\hline $\mathrm{CR}_{3}$ & $\square$ & $\square$ & $\bullet$ & $\square$ & $\square$ & $\square$ & $\diamond$ & $\square$ & $\diamond$ \\
\hline $\mathrm{CR}_{4}$ & $\diamond$ & $\diamond$ & $\diamond$ & $\square$ & $\square$ & $\square$ & $\diamond$ & $\square$ & $\diamond$ \\
\hline $\mathrm{CR}_{5}$ & $\square$ & $\diamond$ & $\square$ & $\square$ & $\square$ & $\diamond$ & $\diamond$ & $\square$ & $\diamond$ \\
\hline $\mathrm{CR}_{6}$ & $\square$ & $\bullet$ & $\square$ & $\square$ & $\square$ & $\square$ & $\square$ & $\diamond$ & $\square$ \\
\hline $\mathrm{CR}_{7}$ & $\square$ & $\square$ & $\square$ & $\square$ & $\square$ & $\diamond$ & $\square$ & $\square$ & $\square$ \\
\hline $\mathrm{CR}_{8}$ & $\square$ & $\diamond$ & $\square$ & $\square$ & $\square$ & $\square$ & $\bullet$ & $\square$ & $\diamond$ \\
\hline $\mathrm{CR}_{9}$ & $\square$ & $\diamond$ & $\diamond$ & $\square$ & $\square$ & $\diamond$ & $\square$ & $\square$ & $\square$ \\
\hline $\mathrm{CR}_{10}$ & $\diamond$ & $\square$ & $\square$ & $\square$ & $\square$ & $\square$ & $\square$ & $\square$ & $\square$ \\
\hline $\mathrm{CR}_{11}$ & $\square$ & 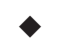 & $\diamond$ & $\square$ & $\square$ & $\square$ & $\square$ & $\diamond$ & $\square$ \\
\hline $\mathrm{CR}_{12}$ & $\square$ & $\diamond$ & $\diamond$ & $\square$ & $\square$ & $\square$ & $\diamond$ & $\diamond$ & $\diamond$ \\
\hline $\mathrm{CR}_{13}$ & $\square$ & $\square$ & $\diamond$ & $\diamond$ & $\diamond$ & $\square$ & $\square$ & $\diamond$ & $\square$ \\
\hline $\mathrm{CR}_{14}$ & $\square$ & $\diamond$ & $\square$ & $\square$ & $\square$ & $\square$ & $\square$ & $\square$ & $\square$ \\
\hline $\mathrm{CR}_{15}$ & $\square$ & $\diamond$ & $\square$ & $\diamond$ & $\square$ & $\square$ & $\square$ & $\square$ & $\square$ \\
\hline $\mathrm{CR}_{16}$ & $\square$ & $\diamond$ & $\square$ & $\diamond$ & $\square$ & $\square$ & $\square$ & $\square$ & $\square$ \\
\hline
\end{tabular}

Notes: $\square$ represents weak; $\diamond$ represents medium; $\diamond$ represents strong.

TABLE 7: Autocorrelation matrix of express SEs.

\begin{tabular}{lcccccccc}
\hline & $\mathrm{SE}_{1}$ & $\mathrm{SE}_{2}$ & $\mathrm{SE}_{3}$ & $\mathrm{SE}_{4}$ & $\mathrm{SE}_{5}$ & $\mathrm{SE}_{6}$ & $\mathrm{SE}_{7}$ & $\mathrm{SE}_{8}$ \\
\hline $\mathrm{SE}_{1}$ & 1 & $\mathrm{H}$ & $\mathrm{L}$ & $\mathrm{VL}$ & $\mathrm{H}$ & $\mathrm{M}$ & $\mathrm{M}$ & $\mathrm{L}$ \\
$\mathrm{SE}_{2}$ & $\mathrm{H}$ & 1 & $\mathrm{M}$ & $\mathrm{L}$ & $\mathrm{VH}$ & $\mathrm{VH}$ & $\mathrm{H}$ & $\mathrm{L}$ \\
$\mathrm{SE}_{3}$ & $\mathrm{~L}$ & $\mathrm{M}$ & 1 & $\mathrm{M}$ & $\mathrm{VH}$ & $\mathrm{L}$ & $\mathrm{H}$ & $\mathrm{M}$ \\
$\mathrm{SE}_{4}$ & $\mathrm{VL}$ & $\mathrm{L}$ & $\mathrm{M}$ & 1 & $\mathrm{~L}$ & $\mathrm{H}$ & $\mathrm{M}$ & $\mathrm{H}$ \\
$\mathrm{SE}_{5}$ & $\mathrm{H}$ & $\mathrm{VH}$ & $\mathrm{VH}$ & $\mathrm{L}$ & 1 & $\mathrm{~L}$ & $\mathrm{M}$ & $\mathrm{M}$ \\
$\mathrm{SE}_{6}$ & $\mathrm{M}$ & $\mathrm{VH}$ & $\mathrm{L}$ & $\mathrm{H}$ & $\mathrm{L}$ & 1 & $\mathrm{H}$ & $\mathrm{M}$ \\
$\mathrm{SE}_{7}$ & $\mathrm{M}$ & $\mathrm{H}$ & $\mathrm{H}$ & $\mathrm{M}$ & $\mathrm{M}$ & $\mathrm{M}$ & 1 & $\mathrm{~L}$ \\
$\mathrm{SE}_{8}$ & $\mathrm{~L}$ & $\mathrm{H}$ & $\mathrm{M}$ & $\mathrm{L}$ & $\mathrm{M}$ & $\mathrm{L}$ & $\mathrm{H}$ & $\mathrm{M}$ \\
$\mathrm{SE}_{9}$ & $\mathrm{~L}$ & $\mathrm{H}$ & $\mathrm{H}$ & $\mathrm{H}$ & $\mathrm{M}$ & $\mathrm{M}$ & $\mathrm{VL}$ & $\mathrm{H}$ \\
\hline
\end{tabular}

Notes: VL: very low; L: low; M: medium; H: high; VH: very high.

brainstorms several SE values options with different realization levels. The normalization for discrete SEs is shown in Table 9.
Considering the constraint of the 1.4 million yuan optimizing budget, the express delivery service decision model for Xi'an SF company is constructed based on formulas 
TABLE 8: House of quality of express service.

\begin{tabular}{|c|c|c|c|c|c|c|c|c|c|c|}
\hline \multicolumn{11}{|l|}{$\overline{S E}$} \\
\hline CR & $\mathrm{SE}_{1}$ & $\mathrm{SE}_{2}$ & $\mathrm{SE}_{3}$ & $\mathrm{SE}_{4}$ & $\mathrm{SE}_{5}$ & $\mathrm{SE}_{6}$ & $\mathrm{SE}_{7}$ & $\mathrm{SE}_{8}$ & $\mathrm{SE}_{9}$ & Weight of CR $\left(w_{i}\right)$ \\
\hline $\mathrm{CR}_{1}$ & 0.1045 & 0.1490 & 0.1127 & 0.0773 & 0.1306 & 0.1101 & 0.109 & 0.1025 & 0.1043 & 0.0685 \\
\hline $\mathrm{CR}_{2}$ & 0.0782 & 0.1236 & 0.1332 & 0.0782 & 0.1486 & 0.0908 & 0.1227 & 0.102 & 0.1227 & 0.0608 \\
\hline $\mathrm{CR}_{3}$ & 0.0683 & 0.1241 & 0.1487 & 0.1006 & 0.1311 & 0.0807 & 0.1166 & 0.1133 & 0.1166 & 0.0442 \\
\hline $\mathrm{CR}_{4}$ & 0.1111 & 0.1432 & 0.1162 & 0.0749 & 0.1316 & 0.1022 & 0.1218 & 0.1088 & 0.0901 & 0.0755 \\
\hline $\mathrm{CR}_{5}$ & 0.0872 & 0.1538 & 0.108 & 0.1084 & 0.0974 & 0.1262 & 0.1038 & 0.1114 & 0.1038 & 0.0689 \\
\hline $\mathrm{CR}_{6}$ & 0.0874 & 0.1477 & 0.0945 & 0.0568 & 0.1238 & 0.1042 & 0.124 & 0.1375 & 0.124 & 0.0674 \\
\hline $\mathrm{CR}_{7}$ & 0.0963 & 0.1684 & 0.0825 & 0.1222 & 0.0846 & 0.155 & 0.1066 & 0.0777 & 0.1066 & 0.0622 \\
\hline $\mathrm{CR}_{8}$ & 0.0835 & 0.1433 & 0.1234 & 0.0970 & 0.1099 & 0.1061 & 0.1046 & 0.1276 & 0.1046 & 0.0755 \\
\hline $\mathrm{CR}_{9}$ & 0.0817 & 0.1344 & 0.1203 & 0.0895 & 0.1334 & 0.1081 & 0.1193 & 0.094 & 0.1193 & 0.0420 \\
\hline $\mathrm{CR}_{10}$ & 0.1591 & 0.1577 & 0.0886 & 0.0524 & 0.1466 & 0.1109 & 0.1146 & 0.0835 & 0.0867 & 0.0689 \\
\hline $\mathrm{CR}_{11}$ & 0.0805 & 0.1316 & 0.1189 & 0.0675 & 0.1415 & 0.0947 & 0.1245 & 0.1165 & 0.1245 & 0.0464 \\
\hline $\mathrm{CR}_{12}$ & 0.0825 & 0.1390 & 0.1164 & 0.0767 & 0.1259 & 0.1007 & 0.1166 & 0.1257 & 0.1166 & 0.0630 \\
\hline $\mathrm{CR}_{13}$ & 0.0721 & 0.1272 & 0.1359 & 0.0959 & 0.1289 & 0.0836 & 0.1194 & 0.1091 & 0.1278 & 0.0755 \\
\hline $\mathrm{CR}_{14}$ & 0.1002 & 0.1454 & 0.0992 & 0.069 & 0.1303 & 0.1215 & 0.1127 & 0.109 & 0.1127 & 0.0689 \\
\hline $\mathrm{CR}_{15}$ & 0.0811 & 0.1270 & 0.1028 & 0.1036 & 0.1151 & 0.1327 & 0.1135 & 0.0983 & 0.1258 & 0.0552 \\
\hline $\mathrm{CR}_{16}$ & 0.0811 & 0.1270 & 0.1028 & 0.1036 & 0.1151 & 0.1327 & 0.1135 & 0.0983 & 0.1258 & 0.0574 \\
\hline Weight of SE $\left(v_{j}\right)$ & 0.0923 & 0.1412 & 0.1121 & 0.0854 & 0.1242 & 0.1103 & 0.1149 & 0.1077 & 0.1123 & \\
\hline Cost $b_{j}$ (ten thousand yuan) & 20.8 & 19.5 & 26.5 & 14.6 & 17.5 & 16.5 & 19.8 & 15.4 & 14.8 & \\
\hline
\end{tabular}

(18)-(25). The objective function is to maximize customer satisfaction.
The details are as follows:

$$
\left\{\begin{array}{l}
\text { MAX }=0.685_{s_{1}}+0.0608_{s_{2}}+0.0442_{s_{3}}+0.0755_{s_{4}}+0.0689_{s_{5}} \\
+0.0674_{s_{6}}+0.0622_{s_{7}}+0.0755_{s_{8}}+0.0420_{s_{9}}+0.0689_{s_{10}} \\
+0.0464_{s_{11}}+0.0630_{s_{12}}+0.0755_{s_{13}}+0.0689_{s_{14}} \\
+0.0552_{s_{15}}+0.0574_{s_{16}} \\
S 1=-1.8141 e^{-y_{1}}+1.0974 \\
S 2=1.1389 y_{2}-0.5104 \\
S 3=-1.7750 e^{-y_{3}}+1.0835 \\
S 4=1.2183 y_{4}-0.5310 \\
S 5=-1.7454 e^{-y_{5}}+1.0471 \\
S 6=1.2028 y_{6}-0.5140 \\
S 7=-1.4611 e^{-y_{7}}+0.8708 \\
S 8=1.4088 y_{8}-0.6993 \\
S 9=0.5642 e^{-y_{9}}-0.8125 \\
S 10=-1.5331 e^{-y_{10}}+0.9180 \\
S 11=1.2198 y_{11}-0.5958 \\
S 12=1.2223 y_{12}-0.5938 \\
S 13=0.5575 e^{-y_{13}}-0.8232 \\
S 16=0.6654 e^{-y_{14}}-0.9927 \\
S 16=0.6654 e^{-y 15}-1.0613 \\
-y_{16}-1.0360 \\
S 5
\end{array}\right.
$$


TABle 9: Normalizations of discrete SEs.

\begin{tabular}{|c|c|c|c|c|c|}
\hline Discrete SEs & Option 1 & Option 2 & Option 3 & Option 4 & Option 5 \\
\hline $\mathrm{SE}_{1}$ & $d_{11}=0.25$ & $d_{12}=0.5$ & $d_{13}=0.8$ & $d_{14}=1$ & - \\
\hline $\mathrm{SE}_{2}$ & $d_{21}=0.2$ & $d_{22}=0.4$ & $d_{23}=0.6$ & $d_{24}=0.8$ & $d_{25}=1$ \\
\hline $\mathrm{SE}_{3}$ & $d_{31}=0.33$ & $d_{32}=0.66$ & $d_{33}=1$ & - & - \\
\hline $\mathrm{SE}_{4}$ & $d_{41}=0.2$ & $d_{42}=0.4$ & $d_{43}=1$ & - & - \\
\hline $\mathrm{SE}_{5}$ & $d_{51}=0.25$ & $d_{52}=0.55$ & $d_{53}=0.75$ & $d_{54}=1$ & - \\
\hline $\mathrm{SE}_{6}$ & $d_{61}=0.25$ & $d_{62}=0.5$ & $d_{63}=0.75$ & $d_{64}=1$ & \\
\hline $\mathrm{SE}_{7}$ & $d_{71}=0.2$ & $d_{72}=0.4$ & $d_{73}=0.6$ & $d_{74}=0.8$ & $d_{75}=1$ \\
\hline $\mathrm{SE}_{8}$ & $d_{81}=0.33$ & $d_{82}=0.66$ & $d_{83}=1$ & - & - \\
\hline $\mathrm{SE}_{9}$ & $d_{91}=0.2$ & $d_{92}=0.4$ & $d_{93}=0.6$ & $d_{94}=0.8$ & $d_{95}=1$ \\
\hline
\end{tabular}

Let $x_{j}$ be the satisfaction level of express service elements. Combined with the data in Table 9, the constraint is

$$
\begin{aligned}
& \left\{\begin{array}{l}
x_{1}=0.25 x_{11}+0.5 x_{12}+0.8 x_{13}+x_{14}, \\
x_{2}=0.2 x_{21}+0.4 x_{22}+0.6 x_{23}+0.8 x_{24}+x_{25}, \\
x_{3}=0.33 x_{31}+0.66 x_{32}+x_{33}, \\
x_{4}=0.2 x_{41}+0.4 x_{42}+x_{43}, \\
x_{5}=0.25 x_{51}+0.55 x_{52}+0.75 x_{53}+x_{54}, \\
x_{6}=0.25 x_{61}+0.5 x_{62}+0.75 x_{63}+x_{64}, \\
x_{7}=0.2 x_{71}+0.4 x_{72}+0.6 x_{73}+0.8 x_{74}+x_{75} \\
x_{8}=0.33 x_{81}+0.66 x_{82}+x_{83}, \\
x_{9}=0.2 x_{91}+0.4 x_{92}+0.6 x_{93}+0.8 x_{94}+x_{95} .
\end{array}\right. \\
& 0<x_{j}<1 \text {. } \\
& x_{11}+x_{12}+x_{13}+x_{14}=1, \\
& x_{21}+x_{22}+x_{23}+x_{24}+x_{25}=1 \text {, } \\
& x_{31}+x_{32}+x_{33}=1 \text {, } \\
& x_{41}+x_{42}+x_{43}=1 \text {, } \\
& x_{51}+x_{52}+x_{53}+x_{54}=1 \text {, } \\
& x_{61}+x_{62}+x_{63}+x_{64}=1 \text {, } \\
& x_{71}+x_{72}+x_{73}+x_{74}+x_{75}=1 \text {, } \\
& x_{81}+x_{82}+x_{83}=1 \text {, } \\
& x_{91}+x_{92}+x_{93}+x_{94}+x_{95}=1 \text {. } \\
& x_{j k} \in[0,1] . \\
& 20.8 x_{1}+19.5 x_{2}+26.5 x_{3}+14.6 x_{4}+17.5 x_{5}+16.5 x_{6}+19.8 x_{7}+15.4 x_{8}+14.8 x_{9} \leq 140 \text {. } \\
& y=R_{i j}^{n o r m} X .
\end{aligned}
$$




$$
\begin{aligned}
R_{i j}^{\text {norm }}= & 0.10450 .14900 .11270 .07730 .13060 .11010 .1090 .10250 .1043 \\
& 0.07820 .12360 .13320 .07820 .14860 .09080 .12270 .1020 .1227 \\
& 0.06830 .12410 .14870 .10060 .13110 .08070 .11660 .11330 .1166 \\
& 0.11110 .14320 .11620 .07490 .13160 .10220 .12180 .10880 .0901 \\
& 0.08720 .15380 .10800 .10840 .09740 .12620 .10380 .11140 .1038 \\
& 0.08740 .14770 .09450 .05680 .12380 .10420 .12400 .13750 .1240 \\
& 0.09630 .16840 .08250 .12220 .08460 .15500 .10660 .07770 .1066 \\
& 0.08350 .14330 .12340 .09700 .10990 .10610 .10460 .12760 .1046 \\
& 0.08170 .13440 .12030 .08950 .13340 .10810 .11930 .09400 .1193 \\
& 0.15910 .15770 .08860 .05240 .14660 .11090 .11460 .08350 .0867 \\
& 0.08050 .13160 .11890 .06750 .14150 .09470 .12450 .11650 .1245 \\
& 0.08250 .13900 .11640 .07670 .12590 .10070 .11660 .12570 .1166 \\
& 0.07210 .12720 .13590 .09590 .12890 .08360 .11940 .10910 .1278 \\
& 0.10020 .14540 .09920 .06900 .13030 .12150 .11270 .10900 .1127 \\
& 0.08110 .12700 .10280 .10360 .11510 .13270 .11350 .09830 .1258 \\
& 0.08110 .12700 .10280 .10360 .11510 .13270 .11350 .09830 .1258
\end{aligned}
$$

The above optimization decision model is processed by Lingo16.0 software. Table 10 lists the solution results about CR achievement level $\left(y_{i}\right)$ and individual customer satisfaction $\left(S_{i}\right)$ by each customer requirement.

The solution results indicate that all the CRs have been achieved at levels greater than 0.85 levels with the 1.4million-yuan budget constraint. The express delivery customer's individual satisfaction $\left(S_{i}\right)$ can be calculated based on the fitting function S-CR. The full $\mathrm{CS}_{i}$ values are obtained from Table 2. When dividing individual customer satisfaction $\left(S_{i}\right)$ by full complete satisfaction $\left(\mathrm{CS}_{i}\right)$, the customer satisfaction level $\left(S_{i} / \mathrm{CS}_{i}\right)$ of each $\mathrm{CR}$ is obtained. According to the objective function in formula (26), when the weight of each customer demand $\left(w_{j}\right)$ is brought in, respectively, the sum of customer satisfaction $(S)$ is 0.4357 and the sum of full customer satisfaction (CS) is 0.5863 , and the customer satisfaction level $(S / C S)$ is $74.31 \%$ in Table 10 . It is worth noting that the full CS for individual CRs is not high in this case, which indicates the full CS rate for the entire service is below $80 \%$. The reason is, according to the defined CS value, if all the responses vote $\mathrm{CR}$ as attractive or one-dimensional and none of them votes for must-be or indifferent requirement, CS values could be equal to 1 . However, this case is uncommon. Moreover, a general product/service essentially has certain must-be requirements, which means some basic customer needs must-be achieved. Especially in the lower price competition express service market, nearly onethird of the requirements among the 16 customer demand categories belong to must-be requirements. Namely, this empirical result is more in line with the actual situation of low-price vicious competition in China's express service market. The presented framework is more objective than traditional methods because the full fulfilment of a certain CR does not ensure customers' full satisfaction. Customers may not need that service element feature. Integrating quantitative Kano's model into FQFD can help firms identify the necessary CRs which significantly affect CS and avoid paying much attention to insignificant customer requirements.

The optimization design scheme of service elements selected also is obtained by Lingo software as follows: $x 13=1, x 25=1, x 32=1, x 43=1, x 54=1, x 64=1, x 74=1$, $x 82=1, x 94=1$. Table 11 shows the results of SE performance and corresponding resource allocation. It can be seen from the results that the fulfilment level of each service element can be determined based on the selected $S E$ points. The actual cost distribution of each service element is obtained by multiplying the SE value of the fulfilment level by the cost index of each service element $b_{j}$, shown in Table 8 .

According to the results of the model in Tables 10 and 11, the capital allocation of 9 service elements, namely, operating standardization of logistics $\left(\mathrm{SE}_{1}\right)$, smooth customer communication channel $\left(\mathrm{SE}_{2}\right)$, personalized service $\left(\mathrm{SE}_{3}\right)$, delivery network coverage (SE4), customer knowledge management $\left(\mathrm{SE}_{5}\right)$, timely update of delivery information $\left(\mathrm{SE}_{6}\right)$, after-sales service management $\left(\mathrm{SE}_{7}\right)$, staff training management $\left(\mathrm{SE}_{8}\right)$, perfect complaint mechanism $\left(\mathrm{SE}_{9}\right)$, is, respectively, 166400 yuan and 195000 yuan, 174900 yuan, 146000 yuan, 175000 yuan, 165000 yuan, 158400 yuan, 101500 yuan, and 118000 yuan. In this case, the highest level of customer satisfaction can be achieved.

\section{Management Inspiration and Research Significance}

5.1. Management Inspiration. Based on the above study, this paper proposes the following management suggestions:

(1) Different service elements have different contributions to customer satisfaction. The proposed method to determine SE optimal values is useful and practical in this paper. When implementing budget allocation, 
TABLE 10: Satisfaction level and customer satisfaction of customer requirement.

\begin{tabular}{lcccc}
\hline $\begin{array}{l}\text { Customer } \\
\text { requirements }\end{array}$ & $\begin{array}{c}\text { CR achievement level } \\
\left(y_{i}\right)\end{array}$ & $\begin{array}{c}\text { Individual customer } \\
\text { satisfaction }\left(S_{i}\right)\end{array}$ & $\begin{array}{c}\text { Full customer satisfaction } \\
\left(\mathrm{CS}_{i}\right)\end{array}$ & $\begin{array}{c}\text { Customer satisfaction level } \\
\left(S_{i} / \mathrm{CS}_{i}\right)\end{array}$ \\
\hline $\mathrm{CR}_{1}$ & 0.8633 & 0.3322 & 0.4300 & 77.27 \\
$\mathrm{CR}_{2}$ & 0.8553 & 0.4637 & 0.6285 & 73.78 \\
$\mathrm{CR}_{3}$ & 0.8506 & 0.3253 & 0.4305 & 75.57 \\
$\mathrm{CR}_{4}$ & 0.8588 & 0.5153 & 0.6873 & 74.97 \\
$\mathrm{CR}_{5}$ & 0.8664 & 0.3133 & 0.4050 & 77.35 \\
$\mathrm{CR}_{6}$ & 0.8539 & 0.5131 & 0.6888 & 74.49 \\
$\mathrm{CR}_{7}$ & 0.8835 & 0.2069 & 0.3333 & 62.08 \\
$\mathrm{CR}_{8}$ & 0.8561 & 0.5068 & 0.7095 & 71.43 \\
$\mathrm{CR}_{9}$ & 0.8631 & 0.5249 & 0.7211 & 72.79 \\
$\mathrm{CR}_{10}$ & 0.2754 & 0.3540 & 77.79 \\
$\mathrm{CR}_{11}$ & 0.4462 & 0.6240 & 71.51 \\
$\mathrm{CR}_{12}$ & 0.8695 & 0.4508 & 0.6285 & 71.73 \\
$\mathrm{CR}_{13}$ & 0.8543 & 0.4847 & 0.6923 & 70.02 \\
$\mathrm{CR}_{14}$ & 0.8546 & 0.5862 & 0.6054 & 96.83 \\
$\mathrm{CR}_{15}$ & 0.8527 & 0.5229 & 0.7474 & 69.96 \\
$\mathrm{CR}_{16}$ & 0.8641 & 0.4911 & 0.7075 & 74.31 \\
Total & 0.8674 & 0.4357 & 0.5863 & \\
\hline
\end{tabular}

TABLE 11: Results of SE fulfilment and optimizing budget allocation.

\begin{tabular}{lccc}
\hline Discrete values of SE & Selected SE options & SE fulfilment level $\left(x_{j}\right)$ & Optimizing budget allocation (ten thousand yuan) \\
\hline $\mathrm{SE}_{1}$ & $x_{13}$ & 0.8 & 16.64 \\
$\mathrm{SE}_{2}$ & $x_{25}$ & 1 & 19.50 \\
$\mathrm{SE}_{3}$ & $x_{32}$ & 0.66 & 17.49 \\
$\mathrm{SE}_{4}$ & $x_{43}$ & 1 & 14.60 \\
$\mathrm{SE}_{5}$ & $x_{54}$ & 1 & 17.50 \\
$\mathrm{SE}_{6}$ & $x_{64}$ & 1 & 16.50 \\
$\mathrm{SE}_{7}$ & $x_{74}$ & 0.8 & 15.84 \\
$\mathrm{SE}_{8}$ & $x_{82}$ & 0.66 & 10.15 \\
$\mathrm{SE}_{9}$ & $x_{94}$ & 0.8 & 11.80 \\
\hline
\end{tabular}

priority should be given to improving the higher importance service elements under the same conditions. That could maximize customer satisfaction and minimize the enterprise's operating cost to the greatest extent. Regarding the empirical study in this paper, Table 8 shows the weight calculation results of service elements. The priority sorting order from high to low is $\mathrm{SE}_{2}-\mathrm{SE}_{5}-\mathrm{SE}_{7}-\mathrm{SE}_{9}-\mathrm{SE}_{3}-\mathrm{SE}_{6}-\mathrm{SE}_{8}-\mathrm{SE}_{1}-\mathrm{SE}_{4}$.

(2) Different types of customer demands have different contributions to customer satisfaction. In service optimization design, enterprises can identify customer demands that significantly affect customer satisfaction by QKANO and avoid excessive efforts on insignificant customer demand. The following are some specific suggestions:

The must-be customer demands $\mathrm{CR}_{1}, \mathrm{CR}_{3}, \mathrm{CR}_{5}$, $\mathrm{CR}_{7}, \mathrm{CR}_{10}$ in this empirical study can be recognized as the basic attributes to win customer satisfaction. If these requirements cannot be achieved, the customer will be highly dissatisfied, so implementing the "must-be" requirements will only create a state of "not dissatisfied" (Matzler and Hinterhuber) [20]. In other words, they are necessary conditions but not sufficient conditions for customer satisfaction (Busacca and Padula) [47].

The one-dimension demand includes $\mathrm{CR}_{2}, \mathrm{CR}_{4}$, $\mathrm{CR}_{6}, \mathrm{CR}_{8}, \mathrm{CR}_{11}, \mathrm{CR}_{12}$ in the study. These demands are more functional to enhance customer satisfaction (Redfern and Davey) [48]. The more these demands are met, the greater the customer satisfaction and vice versa. Therefore, the one-dimension CRs are both the necessary and sufficient conditions (Busacca and Padula) [47]. They are essential in improving service quality and increasing competitive advantage for the enterprise by creating service differences (Witell and Lo"fgren) [17].

The attractive customer requirements, involving $\mathrm{CR}_{9}, \mathrm{CR}_{13}, \mathrm{CR}_{14}, \mathrm{CR}_{15}$, and $\mathrm{CR}_{16}$, are provided to satisfy customers and not lead to dissatisfaction if absent (Berger et al.) [15]. Therefore, customers have no expectations for these service features but will be satisfied when they are properly implemented (Matzler et al.) [19]. So, they are sufficient conditions but not necessary conditions for customer satisfaction (Busacca and Padula) [47]. 
Attractive demand attributes could be portions of the aggressive marketing strategies to attract competitors' customers as much as possible.

5.2. Research Significance. In order to solve the practical issues of express service, this paper constructs an optimization decision-making model for express service based on the quantitative Kano model and FQFD. The priority of express service elements and the allocation of budget funds are determined by solving the model. Compared with the previous research, the most significant advantage of this model is that it fully considers the different contributions of each service element on customer satisfaction and effectively matches customer demands with the service element system. This provides detailed decision supports for express service. The main contributions in theoretical and practical circles are as follows:

(1) This work proposes a measurement method for accurate definition of CRs and CS based on the quantitative Kano model. It not only effectively overcomes the arbitrariness of the traditional qualitative Kano model and improves the objectivity of customer needs' classification but also accurately identifies the relationship function of customer satisfaction and various customer needs.

(2) This study further integrated the triangle fuzzy number into QFD process for the optimization design of express service, which efficiently resolves subjectivity and fuzziness of expert evaluation information. It improves the accuracy of weight determination for customer needs and service elements to enhance further the FQFD model in the express service optimization making-decision support function.

(3) This study constructs a mixed nonlinear programming model of express service optimization decision. The mathematical model takes customer satisfaction as the objective function, considering the budget cost and service elements and their fulfilment level as a series of constraints. Based on the empirical research, this paper recognizes the priority of express service elements from high to low as $\mathrm{SR}_{2}-\mathrm{SR}_{5}-\mathrm{SR}_{7}-\mathrm{SR}_{9}-\mathrm{SR}_{3}$ $\mathrm{SR}_{8}-\mathrm{SR}_{6}-\mathrm{SR}_{1}-\mathrm{SR}_{4}$, while in the budget allocation priority should be given to improving service elements with a higher weight under the same conditions.

5.3. Limitation and Future Research Directions. This study has certain limitations. It focuses on identifying customer requirements, service elements, and their influence on customer satisfaction but ignores the effects of competitive relationships in QFD. Moreover, the research built the optimization model under the economic constraint without considering time and human conditions. The validation of this model is limited to the branches of a well-known Chinese enterprise. Further expanding its verification scope is one of the main directions of further research.
The immediate practical research extension could be taken by collecting data from various sized delivery service companies in China and expanding the customer questionnaires to more groups. Also, competitive relationships between firms should also be considered, and the optimization decision model of express delivery service under the expected competition can be built. Compared with other competitors, enterprises can further define the optimized service elements. Moreover, the subsequent work could advance the optimization model of express service elements under financial, time, and human constraints.

\section{Data Availability}

The data used to support the findings of this study are available from the corresponding author upon request.

\section{Conflicts of Interest}

The authors declare that there are no conflicts of interest regarding the publication of this paper.

\section{Acknowledgments}

The authors are grateful for the supports by the National Social Science Foundation of China (Grant no. 21BJY216), the Humanities and Social Sciences Fund of Ministry of Education in China (Grant no. 19YJA790070), and Major Theoretical and Practical Research Project of Shaanxi Federation of Social Sciences (Grant no. 2021HZ1128).

\section{References}

[1] N. Subramanian, A. Gunasekaran, J. Yu, J. Cheng, and K. Ning, "Customer satisfaction and competitiveness in the Chinese E-retailing: structural equation modeling (SEM) approach to identify the role of quality factors," Expert Systems with Applications, vol. 41, no. 1, pp. 69-80, 2014.

[2] O. Dospinescu, N. Dospinescu, and I. Bostan, "Determinants of e-commerce satisfaction: a comparative study between Romania and Moldova," Kybernetes, 2021.

[3] H. Shan, Q. Tong, J. Shi, and Q. Zhang, "Risk assessment of express delivery service failures in China: an improved failure mode and effects analysis approach," Journal of Theoretical and Applied Electronic Commerce Research, vol. 16, no. 6, pp. 2490-2514, 2021.

[4] S. Vinodh, S. R. Devadasan, and D. Rajanayagam, "The case of implementing innovative total quality function deployment for preventing the sticking of the latching star in electronic switches," Production Planning \& Control, vol. 19, no. 8, pp. 754-769, 2008.

[5] J. Huo and Z. Hong, "Application of service science in the emerging service industries," Service Science in China, pp. 401-461, 2013.

[6] M. Fargnoli and T. Sakao, "Uncovering differences and similarities among quality function deployment-based methods in Design for X: benchmarking in different domains," Quality Engineering, vol. 29, no. 4, pp. 690-712, 2017.

[7] N. Haber and M. Fargnoli, "Prioritizing customer requirements in a product-service system (PSS) context," The TQM Journal, vol. 31, no. 2, pp. 257-273, 2019. 
[8] R. Millen and M. Maggard, "The change in quality practices in logistics: 1995 versus 1991,” Total Quality Management, vol. 8, no. 4, pp. 173-179, 1997.

[9] L. A. Zadeh, "Fuzzy sets as a basis for a theory of possibility," Fuzzy Sets and Systems, vol. 1, no. 1, pp. 3-28, 1978.

[10] L. P. Khoo and N. C. Ho, "Framework of a fuzzy quality function deployment system," International Journal of Production Research, vol. 34, no. 2, pp. 299-311, 1996.

[11] L. K. Chan, H. P. Kao, and M. L. Wu, "Rating the importance of customer needs in quality function deployment by fuzzy and entropy methods," International Journal of Production Research, vol. 37, no. 11, pp. 2499-2518, 1999.

[12] L. V. Vanegas and A. W. Labib, "A Fuzzy Quality Function Deployment (FQFD) model for deriving optimum targets," International Journal of Production Research, vol. 39, no. 1, pp. 99-120, 2001.

[13] S. Lan, H. Zhang, R. Y. Zhong, and G. Q. Huang, “A customer satisfaction evaluation model for logistics services using fuzzy analytic hierarchy process," Industrial Management \& Data Systems, vol. 116, no. 5, pp. 1024-1042, 2016.

[14] N. Haber, M. Fargnoli, and T. Sakao, "Integrating QFD for product-service systems with the Kano model and fuzzy AHP," Total Quality Management and Business Excellence, vol. 31, no. 4, pp. 1-26, 2018.

[15] C. Berger, R. Blauth, and D. Boger, "Kano's method for understanding customer-defined quality," The Center for Quality Management Journal, vol. 2, no. 4, pp. 3-36, 1993.

[16] N. Kano, N. Seraku, F. Takahashi, and S. H. Tsjui, "Attractive quality and must-be quality," Journal of the Japanese Society for Quality Control, vol. 14, no. 2, pp. 147-156, 1984.

[17] L. Witell and M. Lo"fgren, "Classification of quality attributes,” Managing Service Quality, vol. 17, no. 1, pp. 54-73, 2007.

[18] Y. H. Chen and C. T. Su, "A Kano-CKM model for customer knowledge discovery," Total Quality Management, vol. 17, no. 5, pp. 589-608, 2006.

[19] K. Matzler, H. H. Hinterhuber, F. Bailom, and E. Sauerwein, "How to delight your customers," The Journal of Product and Brand Management, vol. 5, no. 2, pp. 6-18, 1996.

[20] K. Matzler and H. H. Hinterhuber, "How to make product development projects more successful by integrating Kano's model of customer satisfaction into quality function deployment," Technovation, vol. 18, no. 1, pp. 25-38, 1998.

[21] C. L. Hsuan and Ko W. Chang, "A fuzzy nonlinear model for quality function deployment considering Kano's concept," Mathematical and Computer Modeling, vol. 48, no. 3-4, pp. 581-593, 2008.

[22] C. Chun and C. M. Chuen, "Integrating the Kano model into a robust design approach to enhance customer satisfaction with product design," International Journal of Production Economics, vol. 114, no. 2, pp. 667-681, 2008.

[23] J. Huiskone and T. Pirttila, "Sharpening Logistics customer service strategy planning by applying Kano's quality element classification," International Journal of Production Economics, vol. 56, pp. 253-260, 1998.

[24] Y. Yoshimitsu, T. Hara, T. Arai, and Y. Shimomura, "An evaluation method for service in the point of customers' view," Proceedings of the 2006 International Conference on Service Systems and Service Management, vol. 1, pp. 7-12, 2006.

[25] M. S. Kim and M. C. Riew, "A strategy for EMSServiceQualityImprovement using KanoModelandImprovement -GapAnalysis," Journal ofKorean Society forQuality Management, vol. 43, no. 3, pp. 397-408, 2015.
[26] Q. Meng, N. Zhou, J. Tian, Y. Chen, and F. Zhou, "Analysis of logistics service attributes based on quantitative Kano model: a case study of express delivering industries in China," Journal of Service Science and Management, vol. 4, no. 1, pp. 42-51, 2011.

[27] M. Qingliang, D. Z. Sasha, and He Lin, "Forecasting service quality dynamics based on integrated method with $\operatorname{GM}(1,1)$ model and Markov chain," Tehnicki Vjesnik-Technical Gazette, vol. 25, no. 5, pp. 1437-1446, 2018.

[28] Z. Li and Q. Meng, "The extended Kano model based on moderated regression analysis: a case study of express delivery service," Journal of Physics: Conference Series, IOP Publishing, vol. 1976, no. 1, , Article ID 012074, 2021.

[29] Y. Akao, Quality Function Deloyment: Integrating Customer Requirements into Product Design, Vol. 21, Productivity Press, , Cambridge, MA, USA, 1990.

[30] F. I. Stuart and S. T. Stephen, "Planning for service quality: an integrative approach," International Journal of Service Industry Management, vol. 7, no. 4, pp. 58-77, 1996.

[31] J. Xiao, X. Wang, and H. Zhang, "Exploring the ordinal classifications of failure modes in the reliability management: an optimization-based consensus model with bounded confidences," Group Decision and Negotiation, pp. 1-32, 2021.

[32] H. Zhang, F. Wang, Y. Dong, F. Chiclana, and E. H. Viedma, "Social trust-driven consensus reaching model with a minimum adjustment feedback mechanism considering assessments-modifications willingness," IEEE Transactions On Fuzzy Systems, vol. 99, p. 1, 2021.

[33] H. Zhang, C.-C. Li, Y. Liu, and Y. Dong, "Modeling personalized individual semantics and consensus in comparative linguistic expression preference relations with self-confidence: an optimization-based approach," IEEE Transactions on Fuzzy Systems, vol. 29, no. 3, pp. 627-640, 2021.

[34] A. Piyanee and Y. Pisal, "Fuzzy QFD approach for managing SCOR performance indicators," Computers \& Industrial Engineering, vol. 122, pp. 189-201, 2018.

[35] E. Bottani and A. Rizzi, "Strategic management of logistics service: a fuzzy QFD approach," International Journal of Production Economics, vol. 103, no. 2, pp. 585-599, 2006.

[36] F. Wang, Li Xu-hong, R. Wei-na, and Y. Zhang, "A fuzzy QFD-based method for customizing positioning of logistics service products of 3PLS," in Proceedings of the International Conference on Wireless Communications IEEE, pp. 3331-3334, Shanghai, China, September 2007.

[37] Y. Lin and S. Pekkkatinen, "QFD-based modular logistics service design," Journal of Business \& Industrial Marketing, vol. 26, no. 5, pp. 344-356, 2011.

[38] K. C. Tan and T. A.. Pawitra, "Integrating SERVQUAL and Kano's model into QFD for service excellence development," Managing Service Quality: International Journal, vol. 11, no. 6, pp. 418-430, 2001.

[39] B. Baki, C. S. Basfirinci, A. R. Llker Murat, and Z. Cilingir, "An application of integrating SERVQUAL and Kano's model into QFD for logistics services: a case study from Turkey," Asia Pacific Journal of Marketing \& Logistics, vol. 21, no. 1, pp. 106-126, 2009.

[40] Y.-E. Nahm, "A novel approach to prioritize customer requirements in QFD based on customer satisfaction function for customer-oriented product design," Journal of Mechanical Science and Technology, vol. 27, no. 12, pp. 3765-3777, 2013.

[41] Y.-E. Nahm, H. Ishikawa, and M. Inoue, "New rating methods to prioritize customer requirements in QFD with incomplete customer preferences," International Journal of Advanced Manufacturing Technology, vol. 65, no. 9, pp. 1587-1604, 2013. 
[42] Z. Fan and L. Zhao, "Integrated quantitative analysis of customer satisfaction based on kano's model," Applied Mechanics And Materials, vol. 224, no. 11, pp. 358-361, 2012.

[43] H. Wang, X. Lu, Y. Du, C. Zhang, R. Sadiq, and Y. Deng, "Fault tree analysis based on TOPSIS and triangular fuzzy number," International Journal of System Assurance Engineering and Management, vol. 8, no. 4, pp. 2064-2070, 2017.

[44] L. H. Chen and M. C. Weng, "A fuzzy model for exploiting quality function deployment," Mathematical and Computer Modeling, vol. 38, no. 5-6, pp. 559-570, 2003.

[45] P. Ji, J. Jin, T. Wang, and Y. Chen, "Quantification and integration of Kano's model into QFD for optimizing product design," International Journal of Production Research, vol. 52, no. 21, pp. 6335-6348, 2014.

[46] E. Gautier and E. Le Pennec, "Adaptive estimation in the nonparametric random coefficients' binary choice model by needlet thresholding," Electronic Journal of Statistics, vol. 12, no. 1, pp. 277-320, 2018.

[47] B. Busacca and G. Padula, "Understanding the relationship between attribute performance and overall satisfaction: theory, measurement and implications," Marketing Intelligence \& Planning, vol. 23, no. 6, pp. 543-561, 2005.

[48] R. Redfern and C. L. Davey, "Supply chain market orientation in new product development in the UK: a pilot case study," Journal of Fashion Marketing and Management, vol. 7, no. 1, pp. 65-77, 2003. 